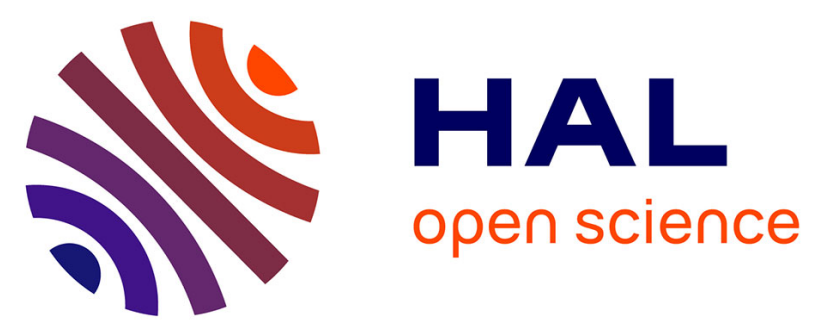

\title{
Paléo-environnement et contrainte fluviale à l'Holocène récent sur les sites de Langoiran et d'Isle-Saint-Georges : bilan de quatre années de recherches géoarchéologiques dans la basse vallée de la Garonne
}

Séverine Lescure, Gilles Arnaud-Fassetta

\section{To cite this version:}

Séverine Lescure, Gilles Arnaud-Fassetta. Paléo-environnement et contrainte fluviale à l'Holocène récent sur les sites de Langoiran et d'Isle-Saint-Georges: bilan de quatre années de recherches géoarchéologiques dans la basse vallée de la Garonne. Aquitania, 2015, 31, p.43-64. hal-01478700

\author{
HAL Id: hal-01478700 \\ https://hal.science/hal-01478700
}

Submitted on 1 Mar 2017

HAL is a multi-disciplinary open access archive for the deposit and dissemination of scientific research documents, whether they are published or not. The documents may come from teaching and research institutions in France or abroad, or from public or private research centers.
L'archive ouverte pluridisciplinaire HAL, est destinée au dépôt et à la diffusion de documents scientifiques de niveau recherche, publiés ou non, émanant des établissements d'enseignement et de recherche français ou étrangers, des laboratoires publics ou privés. 


\section{Paléo-environnement et contrainte fluviale à l'Holocène récent sur les sites de Langoiran et d'Isle- Saint-Georges : bilan de quatre années de recherches géoarchéologiques dans la basse vallée de la Garonne}

\begin{abstract}
RÉSUMÉ
Cette étude participe à la compréhension des dynamiques d'occupation de deux sites archéologiques actuellement présents dans la plaine d'inondation distale de la Garonne maritime : le Castéra médiéval de Langoiran et l'agglomération protohistorique et antique d'Isle-Saint-Georges. Le premier est un castrum implanté au Xl ${ }^{\mathrm{e}}$., "emmoté" le siècle suivant puis abandonné dans le courant du XIV" s. Le second est un habitat groupé du VIII s. a.C. au Ir s. p.C. ayant connu des phases de repli entre le Ive s. et le II ${ }^{e}$ s. a.C. Le fleuve pourrait avoir contribué à cette dynamique d'occupation par le risque (ou du moins la contrainte) qu'il engendre (crues, étiages), les milieux qu'il est susceptible de créer (zones humides) et sa nature dynamique (variabilité géomorphologique latérale, longitudinale, verticale et hydrologique). En outre, il est tout à fait envisageable que, durant l'occupation des sites, le fleuve présentait des caractéristiques hydrographiques (emplacement, nombre de chenaux) et hydrologiques (régime, débit) différentes de celles actuelles. Notre étude vise à éclairer ce contexte paléo-environnemental. Grâce à une approche hydrogéomorphologique mêlant données historiques, archéologiques, géomorphologiques, géophysiques, sédimentologiques, biologiques et chronologiques, nous montrons que les sociétés se sont installées en bordure du fleuve (Isle-Saint-Georges) ou à proximité (Castéra de Langoiran) en profitant probablement des avantages (ressources vivrières, commerce) tirés de cette situation. À Isle-Saint-Georges, la contraction de l'habitat au Second âge du Fer concorde avec la fin de l'activité du paléochenal adjacent. À Langoiran, le site était bordé au XIII ${ }^{\mathrm{e}}$. par une zone humide et et soumis à la fois au risque inondation et au risque d'érosion de la berge proximale. Ces contraintes physiques du milieu peuvent expliquer en partie la dynamique locale de l'habitat, qu'il reste à confronter avec les dynamiques socio-politiques régionales.
\end{abstract}

\section{MOTS-CLÉS}

Garonne maritime, hydrogéomorphologie, géoarchéologie, dynamique fluviale, paléoenvironnements, risque fluvial, interactions Homme/milieu, Holocène, Protohistoire, Antiquité, Moyen Âge

\section{Abstract}

This study contributes to understanding the settlement dynamics of two archaeological sites currently located in the distal flood plain of the tidal Garonne River: the mediaeval Castéra of Langoiran and the protohistoric and antique community of Isle-Saint-Georges. The first one is a castrum constructed in the $11^{\text {th }}$ century, heightened during the $13^{\text {th }}$ century (motte) and abandoned the next century. The second one is a nucleated settlement built between the $8^{\text {th }}$ century BC and the 1st century $\mathrm{AD}$, and retracted between the 4th and the $2^{\text {nd }}$ centuries BC. The Garonne River might have contributed to these settlement changes through the risk generated by flood and low-flow hazards, the environmental potentiality created by wetlands, and lateral, longitudinal and vertical geomorphological dynamics in the floodplain. The Garonne River had different hydrographical (location and number of channels) and hydrological (regime, discharge) characteristics during the sites occupancy. Our study aims to enlighten this paleoenvironmental background. Thanks to a hydrogeomorphological approach mixing historical, archaeological, geomorphological, geophysical, sedimentological, biological, and chronological data, we show that the societies settled down along (Isle-Saint-Georges) or close to the river (Castéra of Langoiran), probably attracted by the advantages of this situation (food supplies, trade). In Isle-SaintGeorges, the contraction of the settlement during the Middle Iron Age agrees with the end of the hydrological activity of the adjacent fluvial channel. At Langoiran, the settlement was closed to a wetland and submitted to the risk of flooding and of erosion of the proximal bank. These natural constraints can explain the local dynamics of the settlements but cannot be completely assessed in the absence of knowledge dealing with the regional, sociopolitical context.

\section{KeYwords}

tidal Garonne River, hydrogeomorphology, geoarchaeology, fluvial dynamics, palaeoenvironments, fluvial risk, humanenvironment interactions, Holocene, protohistory, antiquity, Middle Ages 


\section{INTRODUCTION}

Les sites de Langoiran et d'Isle-Saint-Georges (alt. : 3-6 m NGF) se situent dans le fond de vallée de la Garonne maritime, respectivement à 49 et $41 \mathrm{~km}$ en amont de la confluence de la Dordogne (fig. 1).

Le Castéra de Langoiran (fig. 2A) est une résidence aristocratique fortifiée et fossoyée présente à $400 \mathrm{~m}$ de la rive droite actuelle de la Garonne, sur une éminence topographique de $4600 \mathrm{~m}^{2}$ qui surplombe la plaine alluviale d'1 à 2 m. Les fouilles menées depuis 2004 par S. Faravel (université Bordeaux-Montaigne, Institut Ausonius) ont démontré que cette surélévation était d'origine anthropique (motte) mais nonoriginelle. En effet, alors que le site aurait été implanté au XI ${ }^{\mathrm{e}}$ s., ce n'est qu'au XIII s., lors d'une phase de reconstruction, qu'il aurait été artificiellement remblayé pour être rehaussé. Il aurait ensuite été abandonné (dans le courant du XIV ${ }^{\mathrm{e}} \mathrm{s}$.) au profit de l'actuel château installé $40 \mathrm{~m}$ au-dessus du fond de vallée sur le versant $^{1}$. À Isle-Saint-Georges, les études menées par A. Colin (université Bordeaux-Montaigne, Institut Ausonius) ont mis en exergue un habitat groupé à $350 \mathrm{~m}$ de la rive gauche actuelle de la Garonne. Cet habitat aurait perduré du viII ${ }^{\mathrm{e}}$ s. a.C. au Ir s. p.C. tout en enregistrant des phases de contraction entre le IV s. a.C. et les $\mathrm{II}^{\mathrm{e}} \mathrm{I}^{\mathrm{er}}$ s. a.C. ${ }^{2}$ (fig. $\left.2 \mathrm{~B}\right)$.

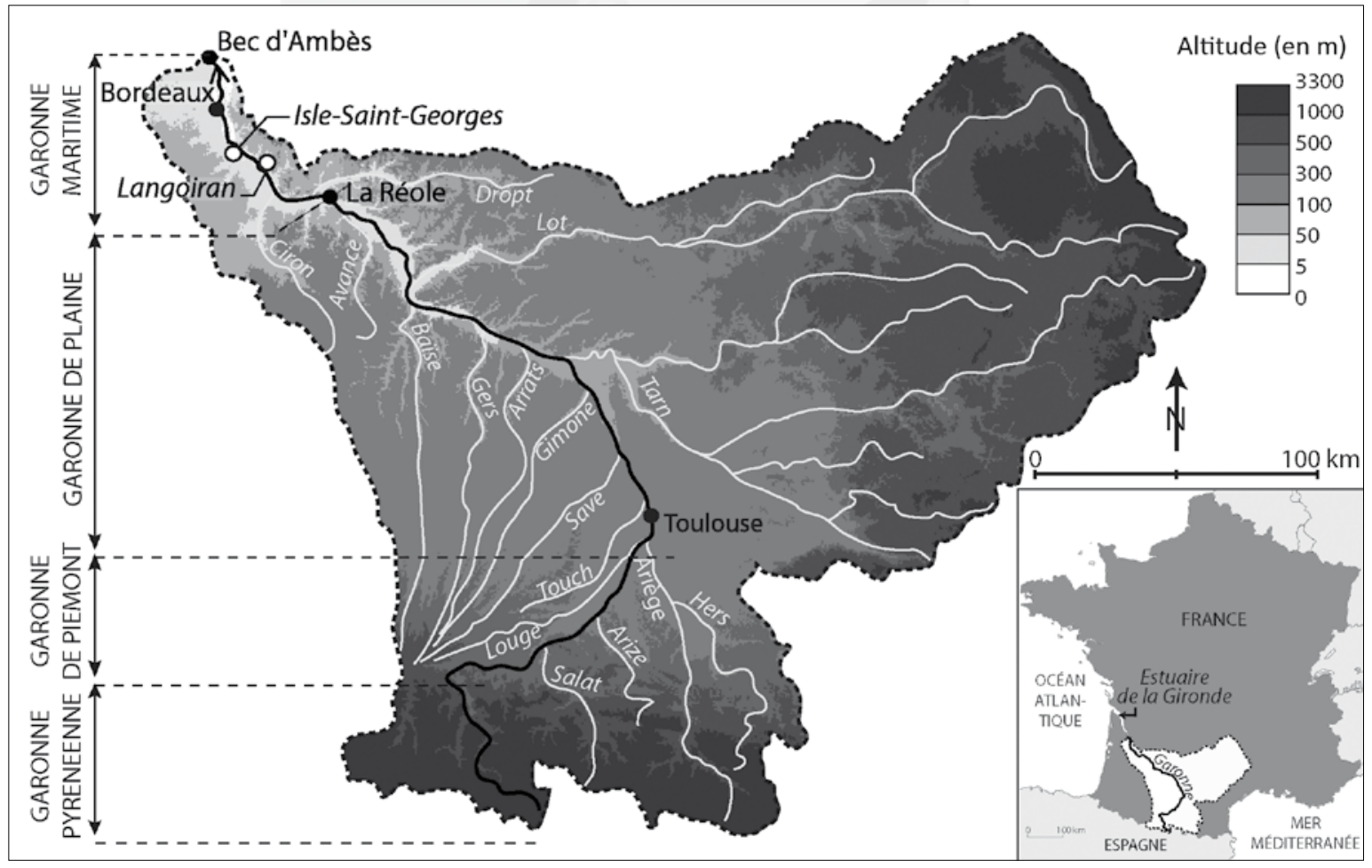

Fig. 1. Localisation de la Garonne maritime et des sites étudiés. (c) Lescure, 2014.

1- Araguas \& Faravel 2006 ; Faravel 2008, 19-50 ; 2010, 69-70 ; 2012, 107-111 ; 2013, 57-60.

2. Colin et al. dans ce volume. 

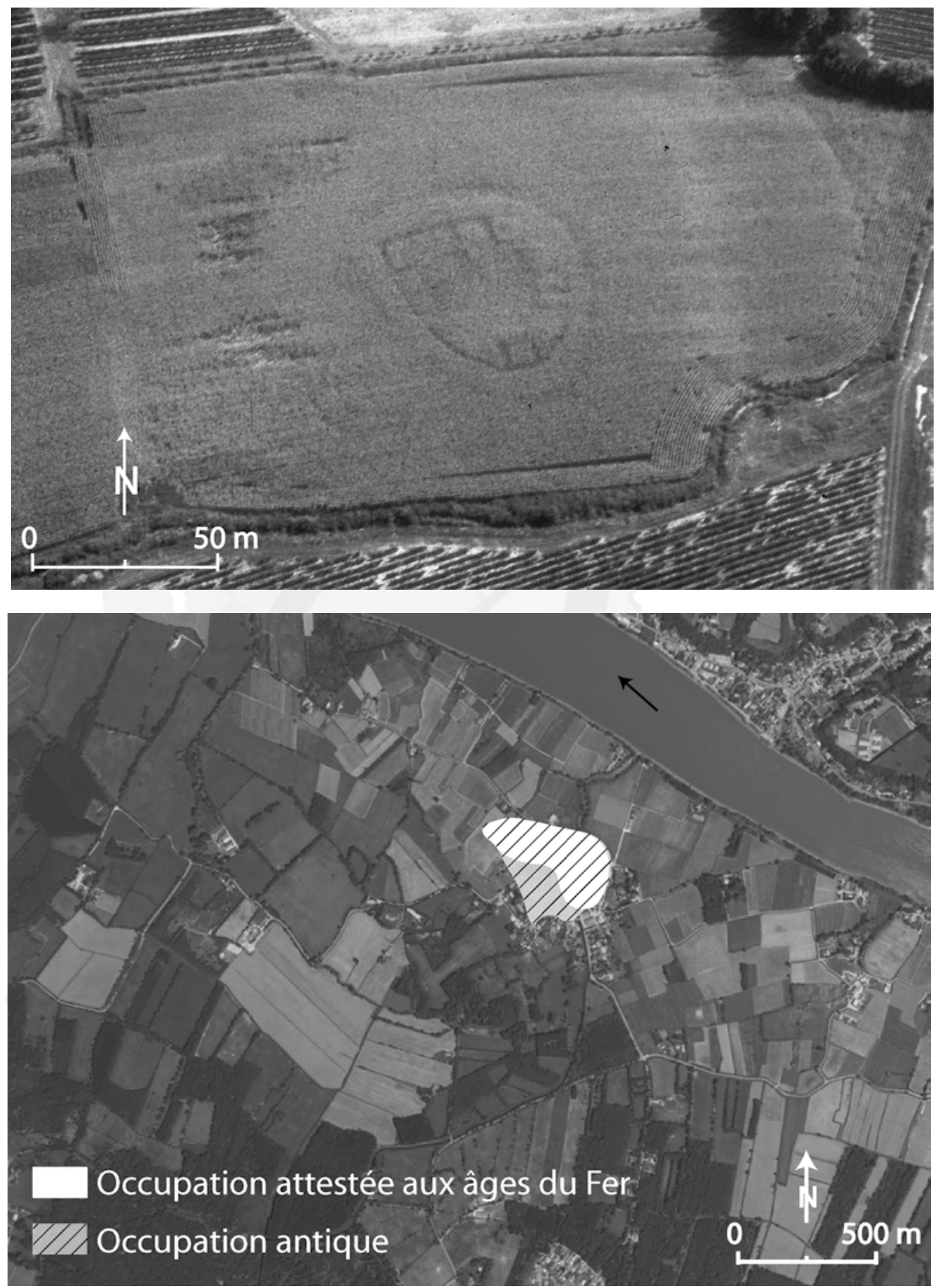

Fig. 2. Sites archéologiques étudiés.

A. Le Castéra de Langoiran. (C) Didierjean, 1987 ;

B. Occupation protohistorique et antique à Isle-Saint-Georges. (c) Lescure, 2014. 


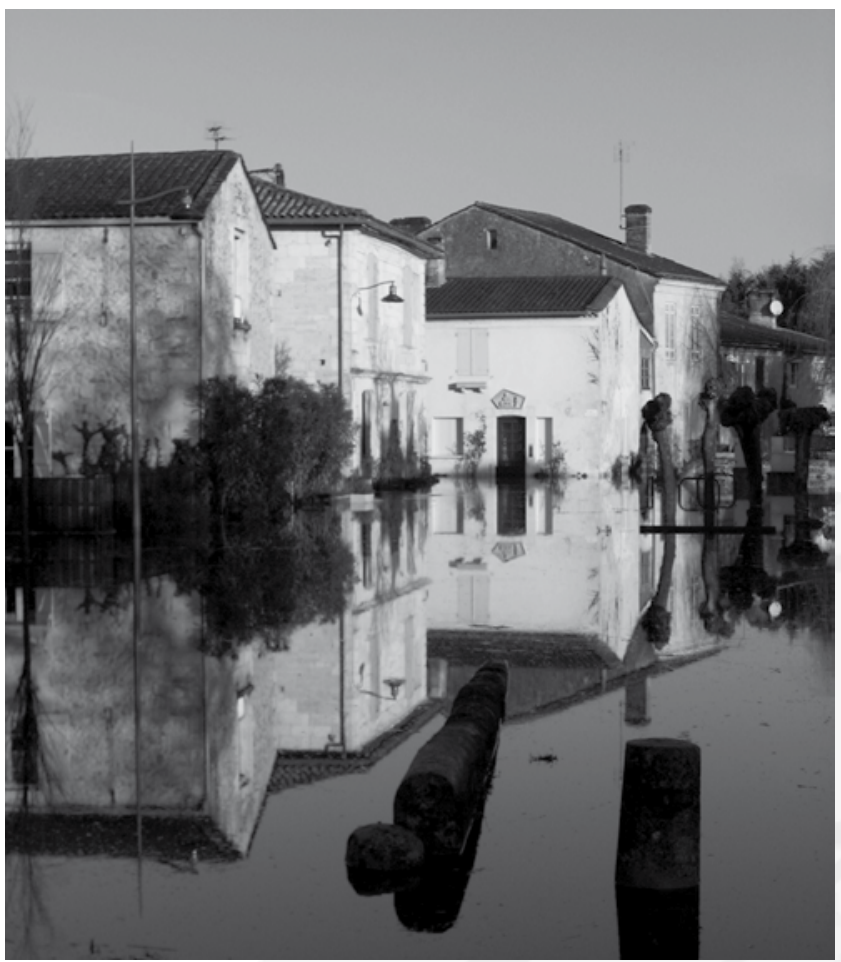

Fig. 3. Inondation du $1^{\text {er }}$ au 3 février 2014 à Isle-Saint-Georges. (C) Mauduit, 2014

L'inondation a été causée par la conjonction de fortes pluies, d'une nappe phréatique sub-affleurante et de forts coefficients de marée qui ont fait déborder la Garonne.
Ces dynamiques d'occupation peuvent relever de contingences historiques et économiques (qui restent cependant indéterminées à ce jour), mais elles soulèvent également la question du rôle du fleuve et, plus généralement, celle de la variable environnementale. Les deux sites étudiés sont actuellement dans la plaine d'inondation de la Garonne maritime : ils sont épisodiquement inondés par les crues du fleuve comme en attestent les événements hydrologiques de février 2014 à Isle-Saint-Georges et de juillet 2014 à Langoiran (fig. 3). Cette contrainte fluviale a-t-elle été de même nature et de même intensité lors de l'occupation protohistorique à médiévale des sites? Cela est peu probable, comme nous allons le démontrer infra.

En effet, les cours d'eau sont des entités en quête permanente d'équilibre, évoluant dans l'espace-temps sous l'effet des variations du débit liquide (Q) ou de la puissance spécifique (w) et du débit solide (Qs) (fig. 4). En cas de modification de ces variables dites de "contrôle", le cours d'eau ajuste sa géométrie pour retrouver un état d'équilibre et de nouvelles conditions de pente, de largeur, de profondeur et de sinuosité (variables dites "de réponse" ${ }^{3}$ ). Plusieurs facteurs sont susceptibles de modifier les débits, directement (via le climat, la géologie, l'eustatisme et, plus récemment, l'Homme) et/ou indirectement (via la couverture végétale). Ainsi, le réchauffement climatique et l'augmentation globale du niveau des mers et des océans depuis le début de la dernière période postglaciaire (i.e., depuis 10000 ans) ont pu considérablement influencer l'évolution de la Garonne maritime (par exemple, modifications de la pente, du tracé en plan, du style fluvial par aggradation), au même titre que les importantes actions humaines entreprises sur l'espace fluvial (comme l'approfondissement du lit par extraction de granulats, ou la stabilisation de sa largeur par endiguement/chenalisation). Un dernier facteur peut avoir engendré des ajustements hydrogéomorphologiques (largeur, profondeur du chenal), il s'agit de la marée. Étant située en amont de l'estuaire, la Garonne maritime est soumise quotidiennement à la marée dynamique qui induit deux fois par jour des variations de hauteur d'eau et de sens du courant (flot/jusant ; jusqu'à $6 \mathrm{~m}$ de marnage) tout en favorisant le transport de matières en suspension (remobilisation des particules fines présentes sur les berges et migration du bouchon vaseux). 


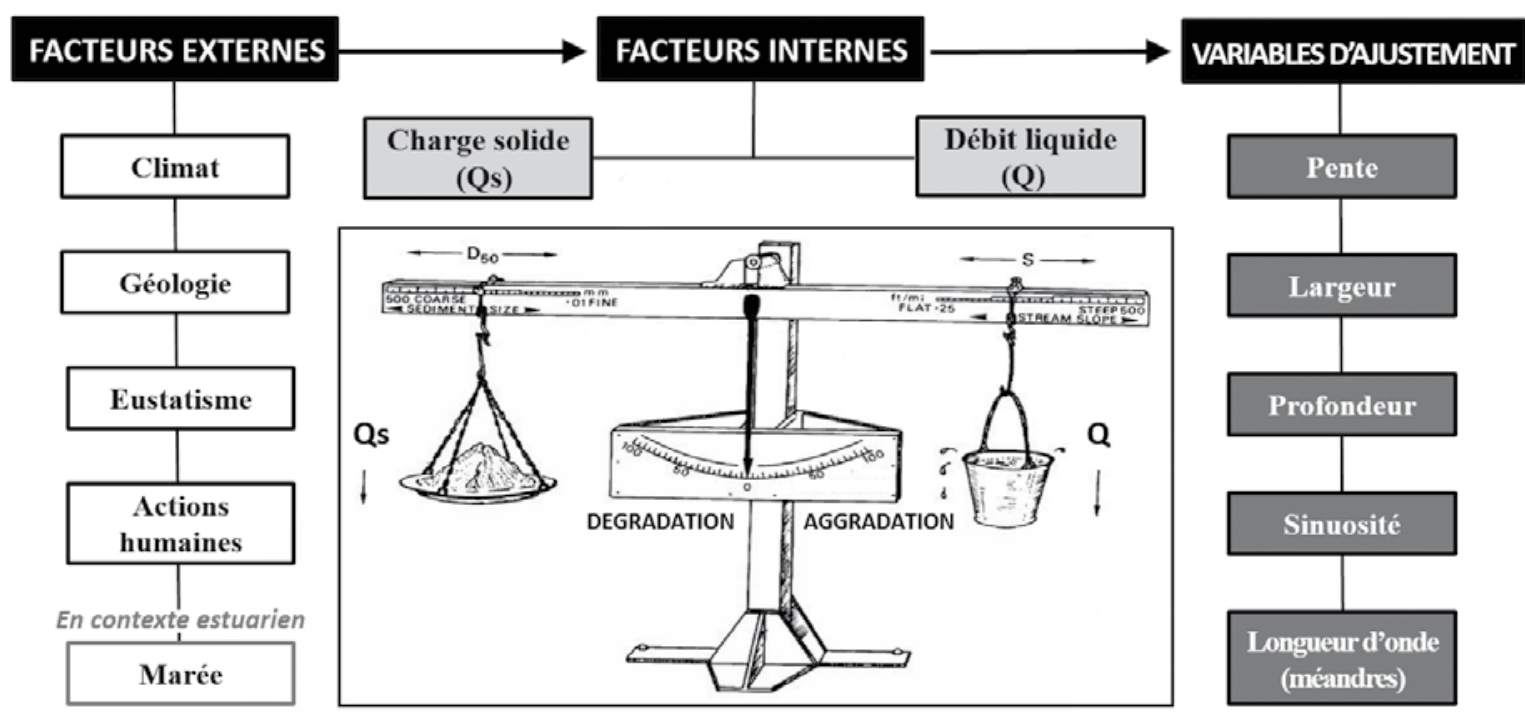

Fig. 4. Facteurs de contrôle de la dynamique fluvio-estuarienne. () Lescure, 2014. "Balance" de Lane, 1955, adapté.

Depuis la fin des années 1980, de nombreuses études ont été menées en France et dans le monde pour rendre compte de l'évolution holocène des hydrosystèmes fluviaux ${ }^{4}$ et fluvio-maritimes ${ }^{5}$. La Garonne fait figure d'exception en étant l'un des seuls grands hydrosystèmes français dont l'histoire des 10000 dernières années est encore peu connue. La Garonne maritime en particulier n'a fait l'objet que de rares études ${ }^{6}$, contrairement à l'estuaire girondin en aval' .

Le présent travail vise à combler cette lacune en reconstituant la dynamique spatio-temporelle holocène de la Garonne dans les secteurs de Langoiran et d'Isle-Saint-Georges (emplacement des différentes unités fluviales, régime du fleuve, intensité et fréquence des crues, environnements associés). Cela permettra d'éclairer le lien entre le milieu fluvial et les sociétés, à la fois en termes d'attractivité (activités économiques liées au fleuve) et/ou de "répulsivité" (inondations et/ou étiages soutenus et/ou fréquents) pour, au final, mieux comprendre et interpréter les dynamiques d'occupation du sol.

\section{CADRE HYDROGÉOMORPHOLOGIQUE}

Le secteur d'étude se situe à égale distance de La Réole, limite amont de la Garonne maritime, et du Bec d'Ambès, point de confluence de la Dordogne où se forme la Gironde (fig. 1).

D'orientation NW-SE et marqué par une dissymétrie transversale, le tronçon de vallée à l'étude est bordé, à l'ouest, par les basses terrasses fluviatiles du Pléistocène moyen (10-50 m d'altitude) et, à l'est, par le plateau calcaire de l'Entre-deux-Mers (50-90 m d'altitude) sur lequel reposent également des vestiges de nappes alluviales d'âge pléistocène (fig. 5). Tout comme l'orientation de la vallée, cette dissymétrie serait

4. Waters 1988 ; Buzzi et al. 1993 ; Qinghai et al. 1996 ; Macklin 1999; Arnaud-Fassetta et al. 2005 ; Castanet 2008 ; Ghilardi et al. 2012 ; Lejeune et al. 2012 ; Vigreux et al. 2012.

5- Malounguila-Nganga et al. 1990 ; Arnaud-Fassetta 1998; Frouin et al. 2010 ; Salomon 2013.

6- $\quad$ Gé et al. 2005 ; Konik et al. 2006.

7. Diot \& Tastet 1995; Pontee et al. 1998 ; Clavé 2001. 


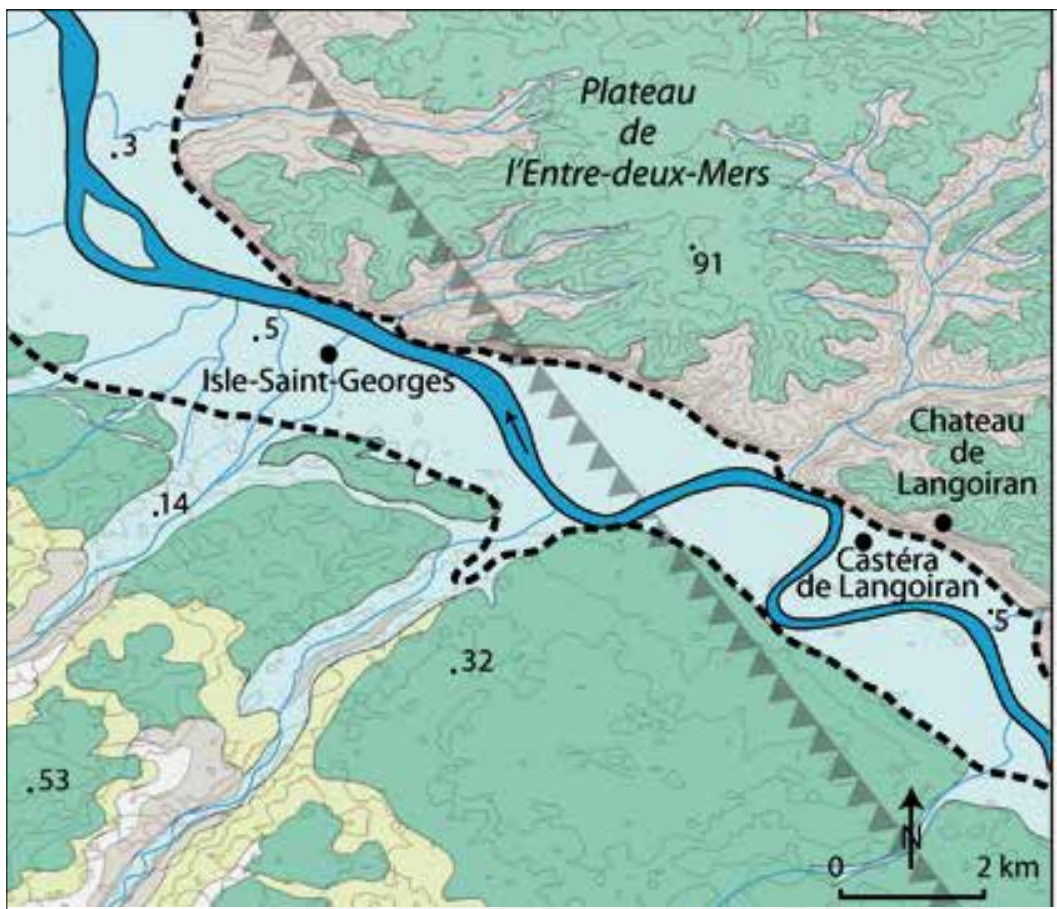

\section{Topographie}

- Courbe de niveau (equidistance $10 \mathrm{~m}$ )

.3 Altitude (en $\mathrm{m}$ )

Castéra Commune ou liev-dit

Hydrologie et hydrographie

$\rightleftarrows$ Lit mineur de la Garonne (et sens d' ecoulement des eaucu)

-2.'. Lit majeur de la Garonne

Petits affluents

Formes et formations fluviatiles

ou éoliennes (Quaternaire)

Terrasses alluviales pleistocènes

Dépots d'origine mixte

(fluviatile et éolienne)

Alluvions holocènes

Formations marines (Tertiaire)

Cakaires oligocènes

Cakaires miocenes

\section{Tectonique}

VVV Faille de Bordeaux (tracé hypothetique)

Fig. 5. Carte géomorphologique du secteur Langoiran-Isle-Saint-Georges. () Lescure, 2014.

héritée de la "flexure celtaquitaine", qui, lors de l'orogénèse hercynienne, a affecté la région selon une ligne Arcachon-Toulouse, accompagnée de la mise en place d'une série de failles de direction NW-SE (comme la faille de Bordeaux, qui passe en rive gauche à Langoiran et en rive droite à Isle-Saint-Georges d'après certains auteurs ${ }^{8}$ ).

Avec un débit moyen compris entre 630 et $700 \mathrm{~m}^{3} / \mathrm{s}$, la Garonne maritime s'écoule dans un chenal unique et globalement subrectiligne (indice de sinuosité de 1,13). Dans le secteur de Langoiran, le fleuve, large de 180-190 m, dessine un méandre d'environ $1730 \mathrm{~m}$ d'amplitude et $2000 \mathrm{~m}$ de longueur d'onde. À Isle-Saint-Georges, le tracé du fleuve devient plus rectiligne ; il s'élargit $(300 \mathrm{~m})$ en embrassant le pied du plateau de l'Entre-deux-Mers sur sa rive gauche.

\section{MÉTHODES D’ÉTUDE}

Pour reconstituer au mieux l'évolution du fond de vallée, nous avons opté pour l'approche multi-données, pleinement adaptée à ce type d'étude géoarchéologique9. Elle croise ici les sources archéologiques et historiques avec les indices géomorphologiques et diverses données issues de prélèvements ou de mesures

8. Cf. la synthèse ANDRA-BRGM sur l'histoire géologique du bassin aquitain, disponible sur : http://sigesaqi.brgm.fr/Structure-ethistoire-simplifiees.html.

9. Bravard et al. 1986 ; Arnaud-Fassetta 2008 ; Salvador et al. 2009 ; Arnaud-Fassetta 2011 ; Arnaud-Fassetta \& Carcaud 2015 
directes sur le terrain : données stratigraphiques (texture granulométrique, structure sédimentaire, perte au feu, susceptibilité magnétique), géophysiques, ostracologiques, palynologiques et chronologiques.

\section{Données archéologiques et historiques}

Les trouvailles archéologiques peuvent renseigner sur la proximité du fleuve : présence de niveaux stériles (sans artefact), structure apparente oblique ou entrecroisée des unités stratigraphiques ou encore présence d'objets (plombs de pêche) ou d'infrastructures (cale à bateaux) liés aux activités fluviales. Mais elles ne suffisent évidemment pas pour déterminer avec précision l'emplacement du réseau hydrographique et l'hydrologie des bras fluviaux. Pour reconstituer l'évolution du système fluvial sur le court terme (Temps modernes), les archives historiques (cartes et textes) se révèlent très utiles, malgré des imprécisions parfois importantes. Dans le cadre de cette étude, sept séries de cartes ont été utilisées : 1) la carte de Matis - 1716 ; 2-3) la carte de Cassini et la carte de Belleyme - seconde moitié du XVIII s. ; 4) la carte d'État-Major - mi$\mathrm{XIX}^{\mathrm{e}}$ s. ; 5) les fonds Billaudel - début XIX ${ }^{\mathrm{e}}$ s. ; 6) le cadastre napoléonien - XIX ${ }^{\mathrm{e}}$ s. ; 7) une carte anonyme de 1868 .

\section{Données géomorphologiques}

Les données géomorphologiques résultent de l'étude de la topographie induite par les dynamiques naturelles et/ou anthropiques. La topographie met en exergue des anomalies du relief pouvant correspondre à de potentiels paléochenaux (anomalie négative), d'anciens bancs alluviaux ou d'anciens cônes de déjection (anomalie positive). Elle est étudiée à partir des cartes topographiques (échelle au 1/25 000), des observations de terrain et de l'exploitation des données LIDAR (Light Detection And Ranging : détection et télémétrie par ondes lumineuses) avec une très haute précision. La morphologie du parcellaire, déterminée à partir d'images satellites, permet aussi d'identifier de possibles paléo-tracés fluviaux qu'il reste alors à confirmer par les données du sous-sol.

\section{Données sédimentologiques}

Celles-ci sont au cœur de l'étude. Quarante et une séquences stratigraphiques ont été analysées sur les sites (fig. $6 \mathrm{~A}$ et C). L'une d'elles provient d'une coupe de 4,7 $\mathrm{m}$ de puissance, ouverte à la pelle mécanique et levée à l'extrémité d'une tranchée archéologique à Isle-Saint-Georges. Trente-trois sont issues de carottes de profondeur "moyenne" (2 à $8 \mathrm{~m}$ ), prélevées avec un carottier portatif à vibro-percussion de type Cobra (Cobra Mk1 du laboratoire PRODIG, Paris, UMR 8586 ; Cobra TT du laboratoire GEODE, Toulouse, UMR 5602) (fig. 6 B). Six autres correspondent à des carottes plus "profondes" ( 8 à 10 m), extraites sous tubes opaques avec un carottier battu sur chenille. Enfin, une séquence provient d'une carotte "complémentaire" de $9 \mathrm{~m}$ de longueur, prélevée sur le site de Langoiran dans des tubes de plastique transparent pour l'analyse palynologique.

Des concordances ont été établies entre chaque unité stratigraphique identifiée afin de déterminer l'étendue des milieux de sédimentation (chenal, levée de berge, delta de rupture de levée, plaine d'inondation) qui se sont succédés dans le fond de vallée. Ce travail a été enrichi par les logs stratigraphiques recensés par le BRGM (5 à Langoiran, 17 à Isle-Saint-Georges).

Des analyses granulométriques ont été réalisées au Laboratoire de Géographie Physique de Meudon (LGP - UMR 8591) sur les échantillons prélevés dans les séquences sédimentaires afin de préciser les caractéristiques des environnements de dépôt (ou milieux de sédimentation) et de l'hydrologie (compétence moyenne et maximale du cours d'eau, modes de transport et de dépôt des sédiments, phases de réactivation/ défluviation/migration des chenaux).

Les séquences les plus épaisses ont fait l'objet d'analyses complémentaires. Des analyses par perte au feu ont été réalisées à la fois sur les six carottes "profondes" et sur la carotte "complémentaire" afin de quantifier 

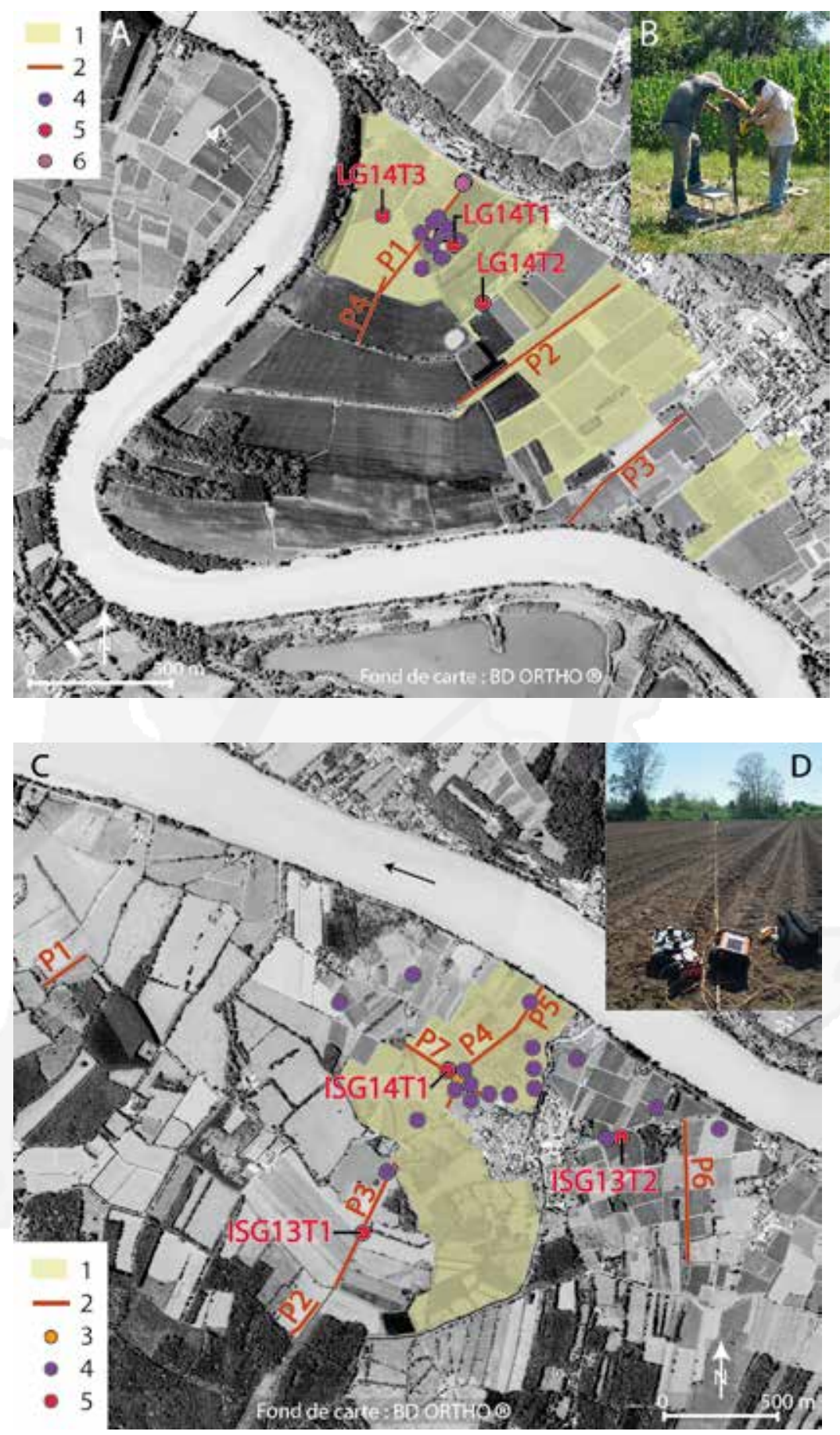

Fig. 6. Synthèse des interventions à Langoiran (A) et à Isle-Saint-Georges (C).

(C) Lescure, 2014 ; B. Carottage avec un Cobra Mk1. (C) Lescure, 2014 ; D. Réalisation d'un profil de résistivité électrique. (c) Lescure, 2014.

1. Emprise des mesures de conductivité électrique apparente du sol, 2 . Profil de résistivité électrique, 3. Coupe stratigraphique de 4,75 $\mathrm{m}$ de puissance, 4 . Séquence verticale de 2 à $8 \mathrm{~m}$ d'épaisseur, 5 . Séquence verticale de 8 à $10 \mathrm{~m}$ d'épaisseur, 6 . Séquence verticale "complémentaire" de 9 m d'épaisseur. 
les teneurs en matière organique ${ }^{10}$. La carotte "complémentaire" a également bénéficié de mesures de la susceptibilité magnétique, à raison d'une mesure par centimètre avec le susceptibilimètre Bartington MS3, afin de détecter la teneur en minéraux ferrimagnétiques des sédiments. L'interprétation de l'évolution verticale de cette teneur s'avère souvent complexe puisqu'elle varie sous l'effet de la nature, de la forme et de la taille des minéraux ${ }^{11}$. Mais couplée avec d'autres paramètres, tels que la granularité et la structure des sédiments, elle peut permettre de distinguer diverses dynamiques environnementales : pédogénèse, érosion des sols ${ }^{12}$, activité hydrologique accrue ${ }^{13}$ ou encore réactivation hydrosédimentaire des chenaux ${ }^{14}$.

\section{Données géophysiques}

Chaque site a fait l'objet de prospections électromagnétiques à large maille à l'aide d'un conductivimètre Geonics EM31 (laboratoire LIENs - UMR 6250), à raison d'environ une mesure/10 $\mathrm{m}^{215}$. Cette technique permet de mesurer la conductivité électrique apparente du sol $(\sigma)$ sur une profondeur de 3 à $6 \mathrm{~m}$ et, ainsi, de détecter puis cartographier les zones humides et les paléochenaux. Réalisées avant les prélèvements in situ, ces prospections ont été croisées avec les données géomorphologiques pour choisir l'emplacement des carottages.

De plus, des profils de tomographie de résistivité électrique (ERT) ont été réalisés perpendiculairement au chenal actuel de la Garonne avec un résistivimètre ABEM Terrameter LS (LGP ; fig. 6 D). Les profils ERT ont atteint une profondeur maximale de $35 \mathrm{~m}$ à Isle-Saint-Georges (espacement inter-électrodes : $3 \mathrm{~m}$ ) et de $13 \mathrm{~m}$ à Langoiran (espacement inter-électrodes : $2 \mathrm{~m}$ ). Cette technique permet d'acquérir le même type de données - la résistivité électrique est l'inverse de la conductivité - mais dans le plan horizontal et non vertical. C'est ainsi une très bonne technique pour obtenir la géométrie des paléochenaux qui, croisée aux données sédimentologiques (texture, structure), permet d'établir des quantifications hydrauliques (débit, puissance fluviale) et hydrosédimentaires (compétence, capacité de transport) ${ }^{16}$.

\section{Données ostracologiques}

Des analyses ostracologiques sont en cours ${ }^{17}$ sur les huit carottes dont la longueur atteint ou dépasse $8 \mathrm{~m}$ de longueur. Les ostracodes sont des arthropodes bivalves dépassant rarement la taille millimétrique, qui vivent dans des milieux humides variés selon les espèces (océan, fleuve, marais...). L'étude de la répartition des ostracodes fossiles au sein des séquences sédimentaires (espèce, stade d'évolution des individus, valves entières ou cassées) fournit donc de précieuses informations sur la qualité des eaux (douce, saumâtre, salée) et l'énergie du point d'eau (courant fort ou faible, eau stagnante) ${ }^{18}$.

\section{Données palynologiques}

La carotte "complémentaire" a fait l'objet d'une étude palynologique par É. Faure (laboratoire GEODE). Sur les $9 \mathrm{~m}$ de la séquence, 39 échantillons ont été analysés entre +1,6 et -3 m NGF (séquences considérées comme pertinentes en raison de leur richesse en matière organique). L'objectif était d'avoir un signal paléo-

10. Dean 1974 ; Heiri 2001.

11. Thompson \& Morton 1979.

12. Dearing et al. 1996.

13. Arnaud et al. 2005.

14. Castanet et al. 2007.

15- Cf. article de Mathé \& Camus dans ce volume.

16- Ce travail de quantification est en cours de réalisation sur le secteur étudié. Il a donné des résultats très probants dans d'autres hydrosystèmes tels que le delta du Rhône : Arnaud-Fassetta 2008, 5-6.

17- Ces analyses ont été effectuées par S. Lescure et contrôlées par M. E. Montenegro.

18. Whatley 1988. 
écologique qui permette à la fois de renseigner les paléoenvironnements (mise en place d'une zone humide, asséchement/ouverture du milieu) et les activités humaines (par exemple, indices de mise en valeur agricole).

\section{Données chronologiques}

Les données chronologiques sont indispensables pour fournir un cadre temporel à l'étude et pour calculer les taux de sédimentation, qui sont de précieux indicateurs de la dynamique fluviale (comme l'accroissement du taux de sédimentation en cas d'une augmentation du nombre et/ou de l'intensité des crues). Trois sources ont été utilisées : les niveaux archéologiques, les datations radiocarbones et les datations par luminescence optiquement stimulée (OSL). Vingt-et-une datations radiocarbone AMS ont été réalisées par Beta Analytic et deux autres auprès du laboratoire de Hanovre. Cependant, quatre d'entre elles n'ont pu être exploitées suite à des problèmes d'inversion de dates dans la séquence ou de dates faussement contemporaines (probablement causés par un remaniement du matériel lors du prélèvement). Tous les âges conventionnels obtenus (en BP) ont été calibrés à l'aide du logiciel dédié OxCal 4.2 ${ }^{19}$. Dix autres échantillons provenant des carottages "profonds" sont en cours de datation par la méthode OSL à l'IRAMAT-CRP2A (Bordeaux, UMR 5060).

\section{RÉSULTATS}

Les résultats obtenus permettent, dans un premier temps, de suivre l'évolution latérale du lit de la Garonne depuis le VI e millénaire a.C. et, dans un second temps, d'éclairer les relations Homme/milieu fluvial.

\section{Évolution du réseau hydrographique depuis le Mésolithique}

\section{De la fin du Mésolithique au Néolithique}

Au Mésolithique final et au début du Néolithique, la Garonne occupait un espace bien différent de l'actuel : le fleuve passait à plusieurs centaines de mètres de la rive droite actuelle à Langoiran et se trouvait à plus d'1 km en rive gauche vers Isle-Saint-Georges (fig. $7 \mathrm{~A}$ et B). La Garonne était alors bien plus large qu'aujourd'hui (500 à $720 \mathrm{~m}$ d'après les profils ERT) et comportait un (style à méandres) ou plusieurs bras (style divagant) d'écoulement. Plusieurs scénarios sont envisageables.

Toutes les données attestent l'existence de cet ancien cours du Mésolithique final/début du Néolithique dans les deux secteurs étudiés : les parcelles sont alignées sur le tracé supposé, la conductivité électrique est forte ${ }^{20}$ et, surtout, de la grave, charge caractéristique du fond d'un chenal relativement énergique, est présente

en profondeur. À Isle-Saint-Georges, la grave précède un niveau sableux daté de 5718-5611 a.C. puis des dépôts limoneux organiques (fig. 8). À Langoiran, la grave n'a pas été atteinte mais on retrouve systématiquement cette unité sableuse (datée de 6217-6066 a.C. à sa base, 5558-5471 a.C. à son sommet) surmontée de dépôts organiques ( 5550 à 3300 a.C.) (fig. 9). Ces derniers ont été formés en contexte de zone humide à eaux stagnantes comme l'atteste l'étude palynologique (présence notamment de Mougeotia, algues de la famille des Zygnématacées) et l'étude sédimentologique (perte au feu, granulométrie, susceptibilité magnétique) de la séquence "complémentaire" prélevée à Langoiran (niveaux riches en matière organique ; fig. 10). On démontre ainsi que la mise en place de la zone humide fait suite à plusieurs siècles de variabilité

19. Bronk Ramsey 1995.

20. Camus \& Mathé, dans ce volume. 
Fig. 7. Évolution holocène supposée de la Garonne à Langoiran ( $\mathrm{A}$ ) et à Isle-Saint-Georges (B). (C) Lescure, 2014.

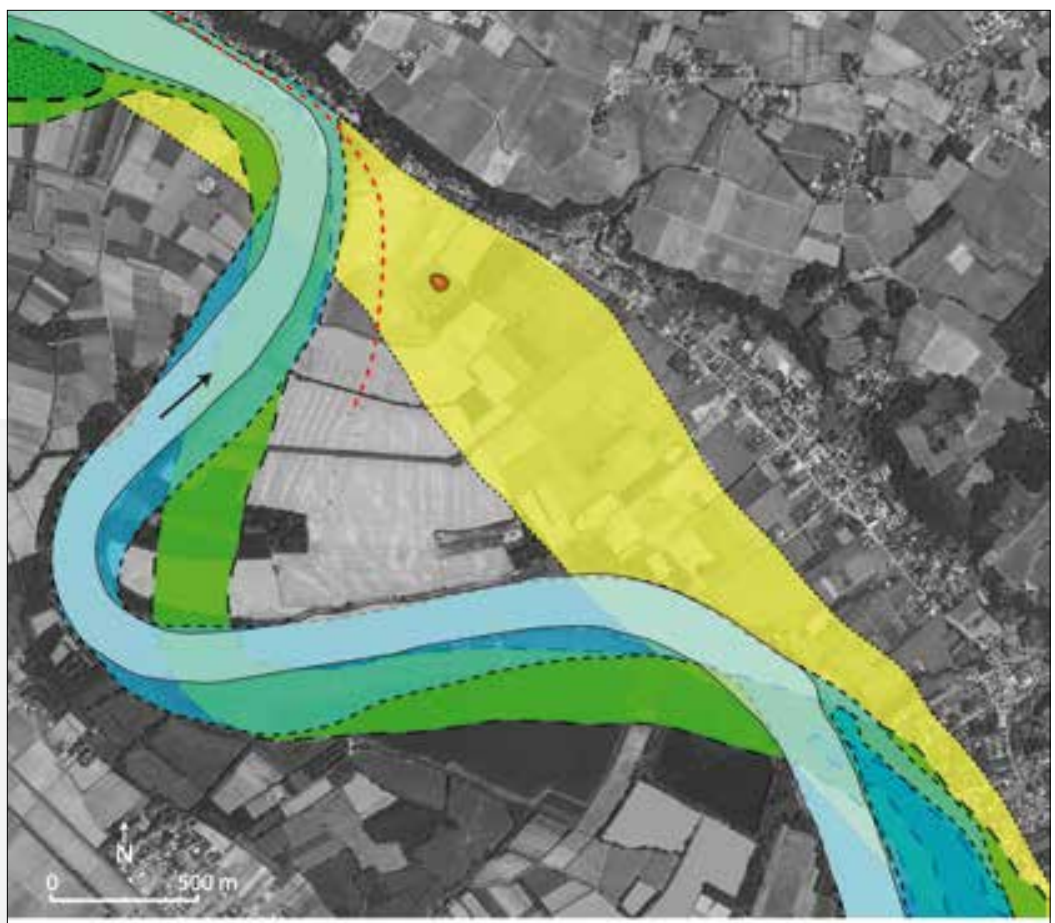

\section{$\begin{array}{lll}\text { 1) Antérieur à Poccupation } & \text { 2) Contemporain aे l'occupation 3) Post-occupation }\end{array}$}

:- jLa Garonne au Mesolithique final.

Emprise maximale potentiolle Presence ofun ou plusieurs bros d'écoulement. Mise en evidence

- Costrum de Langoiran occupation estimée du Xule a mi-Xille siecle. marécogeux du deburt du Nélofi-

- Berge orientale potentielle de la Garonne

?]

tid Chenal et iles au Xvilo siècle

ind Chenal et lles dans la seconde i.s moitié du XIXe siecle thique ou pled du versant.

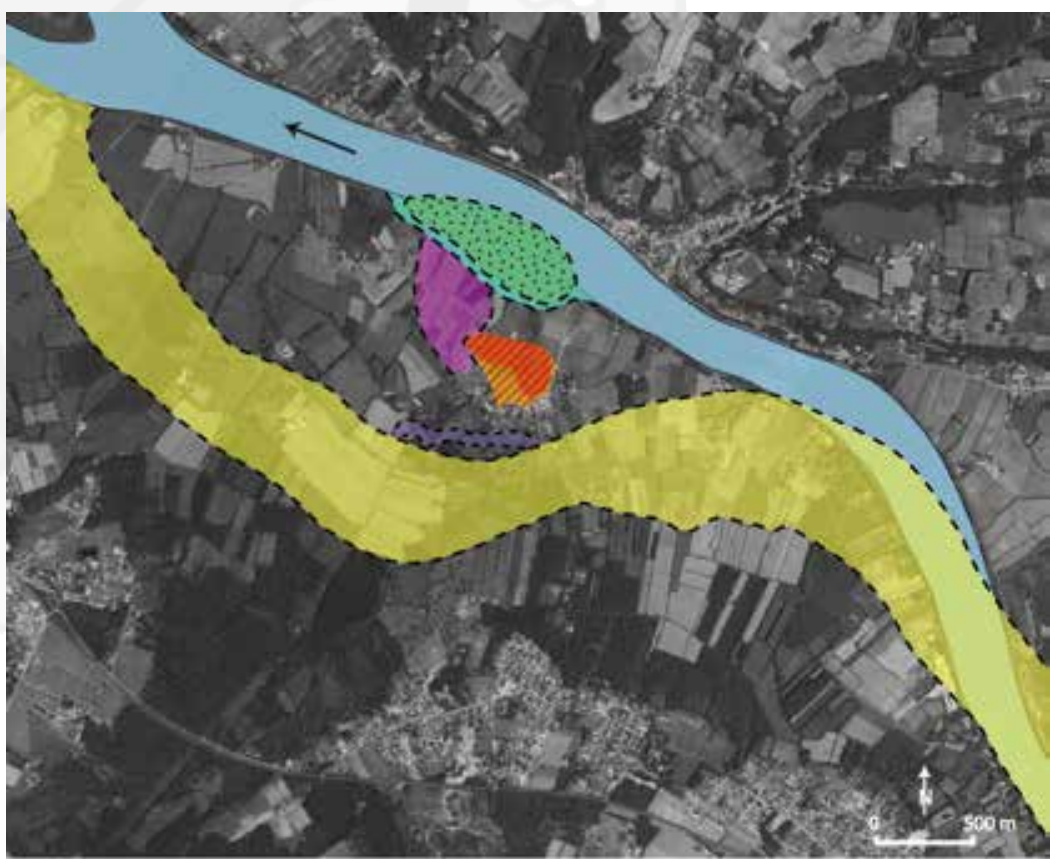

1) Antérieur à l'occupation

$1^{--I}$ La Garonne au Mèsolithique

Emprise maximale potentielle. Presence dun ou plusieurs bras decoulement. Mise en evidence dune evolution en bras-mort markecageux ou debut du Netolithique.

\section{2) Contemporain à Poccupation}

Occupation attestée aux àges du Fer 108 Occupation antique

-3 Portion dun chenal potentiellement actif aux jusqu'aux âges du Fer.

Portion dun chenal secondaire poten tiellement actif jusqu'au début du Moyen Age.
3) Post-occupation

E-3 Chenal et ile Dig au XIIr siecle

Chenal actuel de la Garonne 


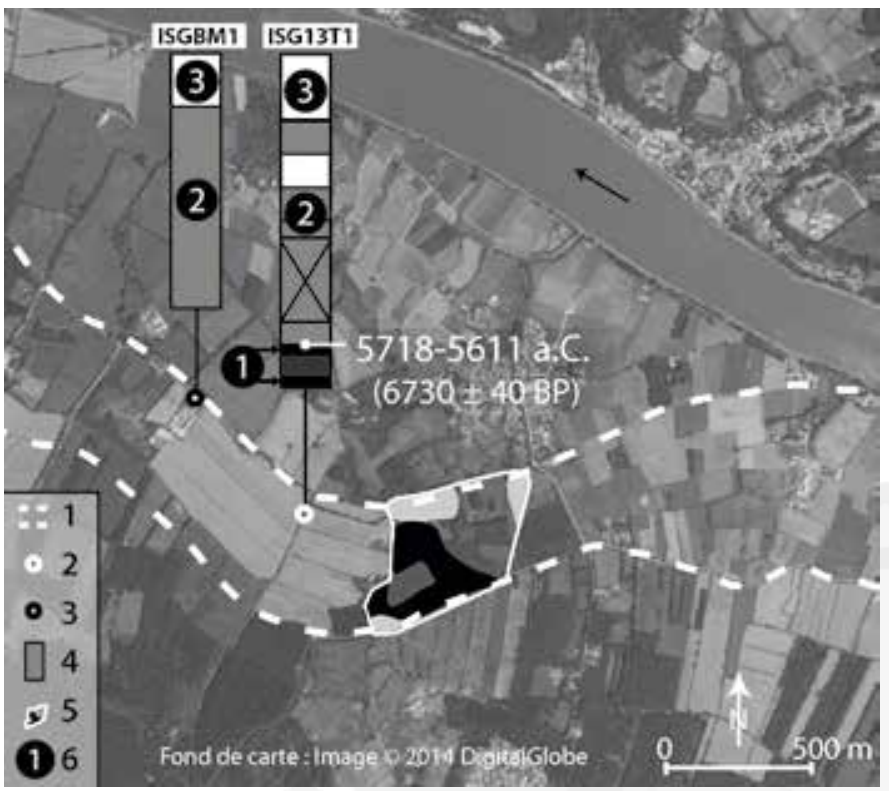

Fig. 8. Mise en évidence d'un paléochenal du début du Néolithique : I'exemple d'Isle-Saint-Georges. (c) Lescure, 2014.

1. Limites supposées, déterminées à partir de la morphologie du parcellaire et des profils de résistivité électrique ; 2 . Carotte "profonde" de $10 \mathrm{~m} ; 3$. Log du BRGM de $7,70 \mathrm{~m} ; 4$. Séquence stratigraphique avec, en blanc: argiles; en gris foncé : vase ou argiles riches en matière organique; en noir : sables grossiers ou grave; croix : hiatus; 5 . Emprise des prospections géophysiques avec, en gris clair : conductivité $<60$ à $\mathrm{mS} / \mathrm{m}$; en noir : conductivité entre 60 et $80 \mathrm{mS} / \mathrm{m}$.

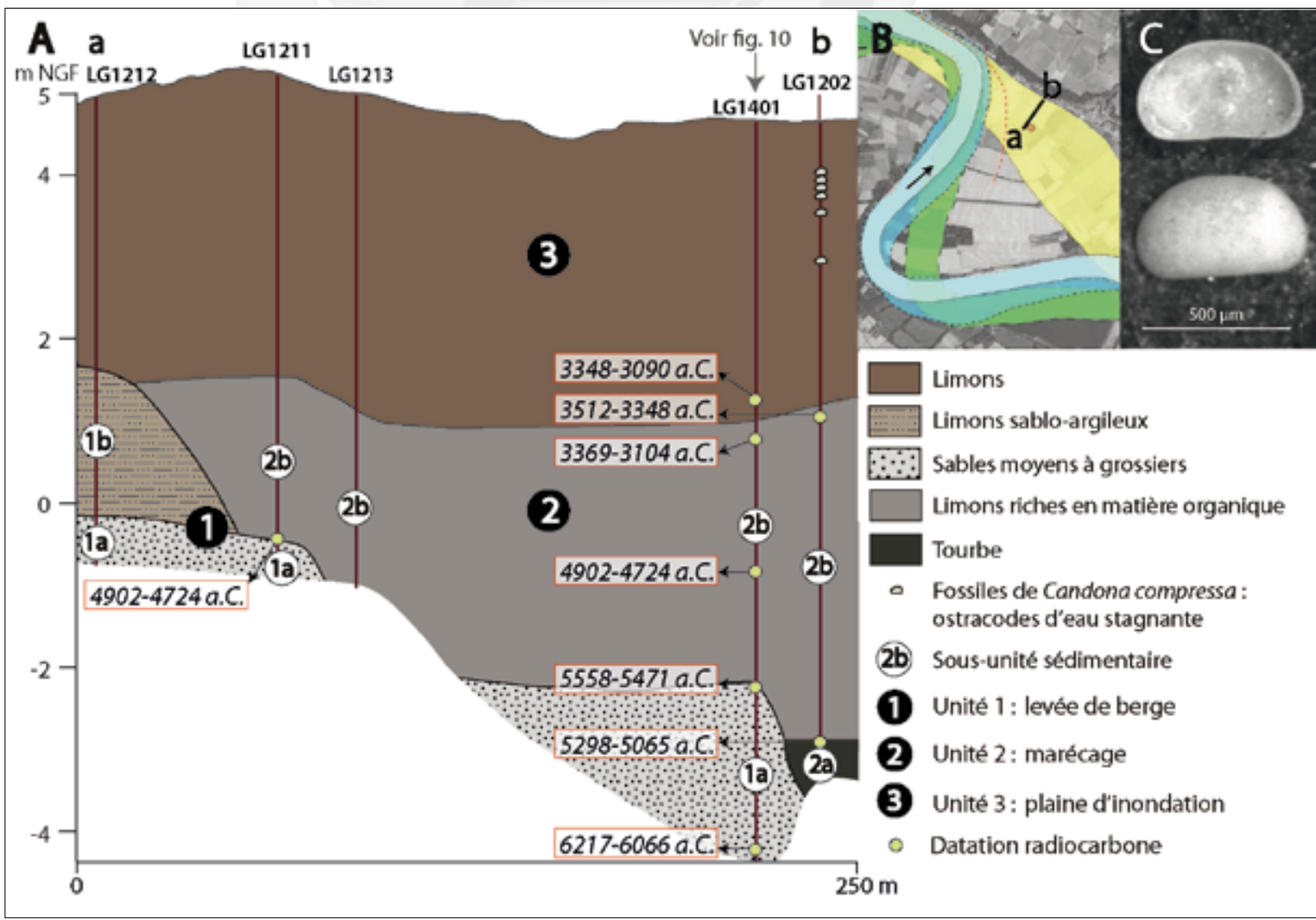

Fig. 9. Transect stratigraphique à travers le bras-mort néolithique de Langoiran. (C) Lescure, 2014.

A. Transect stratigraphique ; B. Localisation du transect ; C. Vue interne (en haut) et externe (en bas) de valves de Candona aff. compressa. Aucun vestige archéologique n'a été retrouvé dans ces sondages mais, juste en amont, les niveaux d'occupation du Castéra se trouvent entre $+4,5$ et +6 m NGF. 


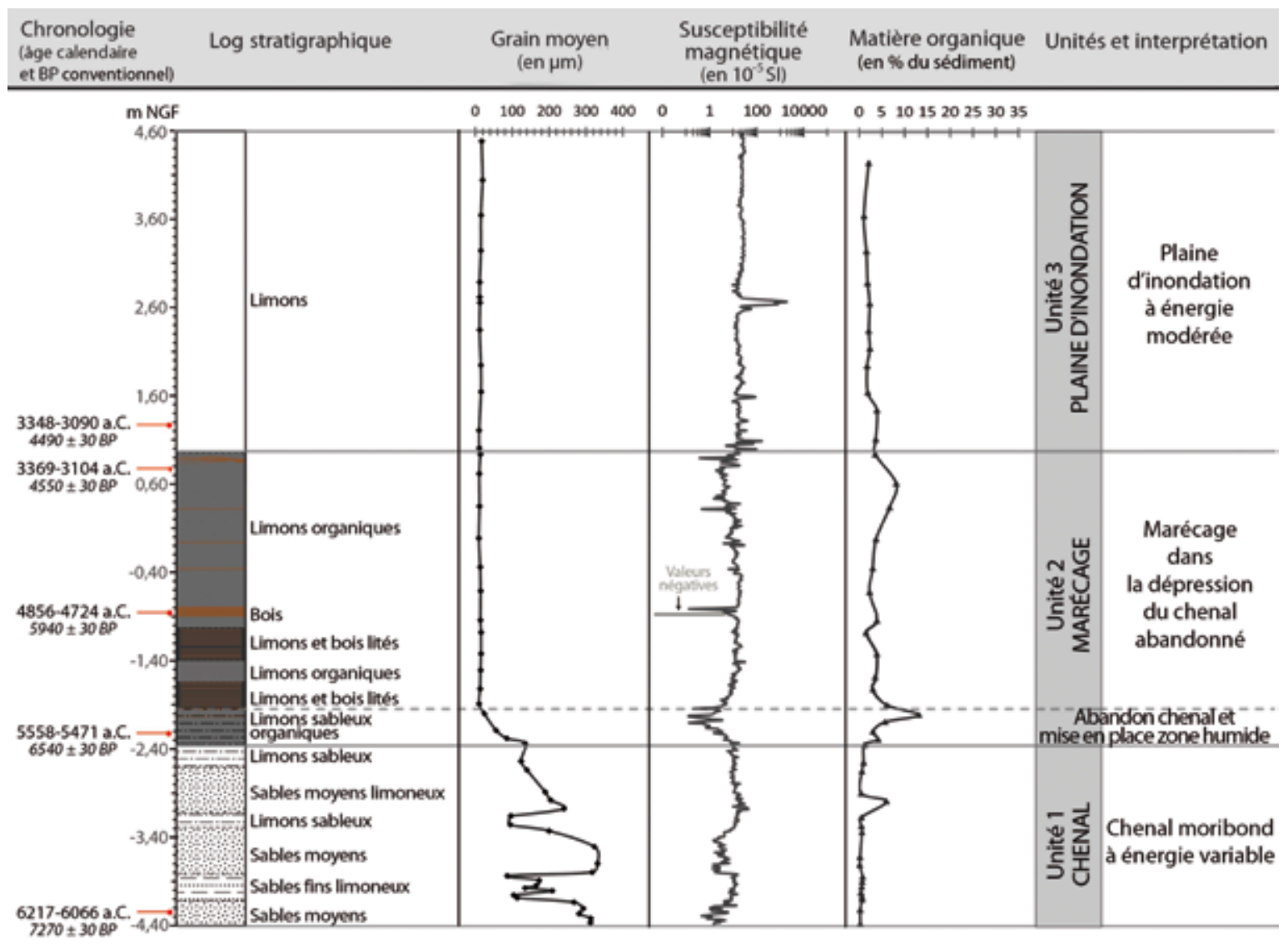

Fig. 10. Analyse sédimentologique de la séquence LG401 prélevée dans le bras-mort néolithique. (c) Lescure, 2014. Les valeurs basses de la susceptibilité magnétique reflètent ici la richesse en bois et en matière organique diffuse dans les sédiments. Cette richesse atteste la mise en place d'un marécage dans la dépression du chenal abandonné vers 5558-5471 a.C. (fig. 9) puis son asséchement vers 3300-3100 a.C. au profit d'une plaine d'inondation moins humide, mais néanmoins régulièrement submergée, comme en témoigne la présence d'ostracodes vivant en eau douce stagnante (l'étude est en cours, mais les premiers résultats confirment la présence de Candona compressa dans les premiers mètres, comme dans la carotte LG1202).

hydrologique (alternance de niveaux sableux et limoneux-sableux associés à des écoulements dont la compétence moyenne varie de 86 à $331 \mu \mathrm{m}$ entre 6217-6066 et 5558-5471 a.C.).

Localement, la stratigraphie atteste une déperdition de l'énergie du fleuve au Mésolithique final (passage des galets aux sables), suivie d'une instabilité hydrologique (alternance sables/limons sableux) au début du Néolithique puis de l'abandon du chenal qui devient alors un bras-mort marécageux. Cet abandon peut être dû soit à un changement de bras fluvial (par défluviation), soit à un changement de style fluvial [passage du style divagant à chenaux multiples ("wandering pattern") au style sinueux à chenal unique]. En tout cas, il est très probable que tous ces changements opérés depuis 8000 ans soient liés à la hausse du niveau marin connexe au réchauffement global holocène. En effet, la remontée du plan d'eau océanique a provoqué une diminution de la pente et une réduction de la puissance fluviale, conduisant à des changements de tracé et à l'abandon d'un ou plusieurs bras. La variation de la fourniture sédimentaire en provenance du bassinversant, sous contrôle de la variabilité hydroclimatique et de l'anthropisation (via la couverture végétale qui 
protège de l'érosion), peut également être un facteur de contrôle, de même que l'activité tectonique. Un affaissement en rive gauche du bloc inférieur de la faille de Bordeaux aurait pu conduire de facto au déplacement de la Garonne en direction de la ligne de faille à Isle-Saint-Georges (qui se trouve sur ce même bloc).

Les profils de résistivité électrique ne permettent pas de privilégier l'une ou l'autre des hypothèses (défluviation ou changement de style fluvial) puisqu'ils démontrent la présence de plusieurs "sillons" dans l'espace considéré, ce qui rend particulièrement complexe la délimitation du lit fluvial. Sur les deux sites, une ou deux berges "principales" peuvent être distinguées grâce à une augmentation importante de la résistivité. Entre ces berges, la résistivité s'effondre localement sur plusieurs mètres de profondeur à deux voire trois reprises selon le profil (fig. 11). En considérant que ces sillons correspondent à des paléochenaux, on envisage deux scénarios : ces paléochenaux sont 1) diachrones ou 2) synchrones.

Dans le scénario 1, il est probable que les paléochenaux les plus profonds, larges d'une centaine de mètres, datent de la dernière glaciation (niveau marin jusqu'à 120-130 m plus bas que l'actuel au Dernier Maximum Glaciaire, il y a environ 20000 ans), avant que le plan d'eau océanique ne s'élève pour atteindre son niveau actuel; les paléochenaux les moins profonds sont datés du Mésolithique/Néolithique.

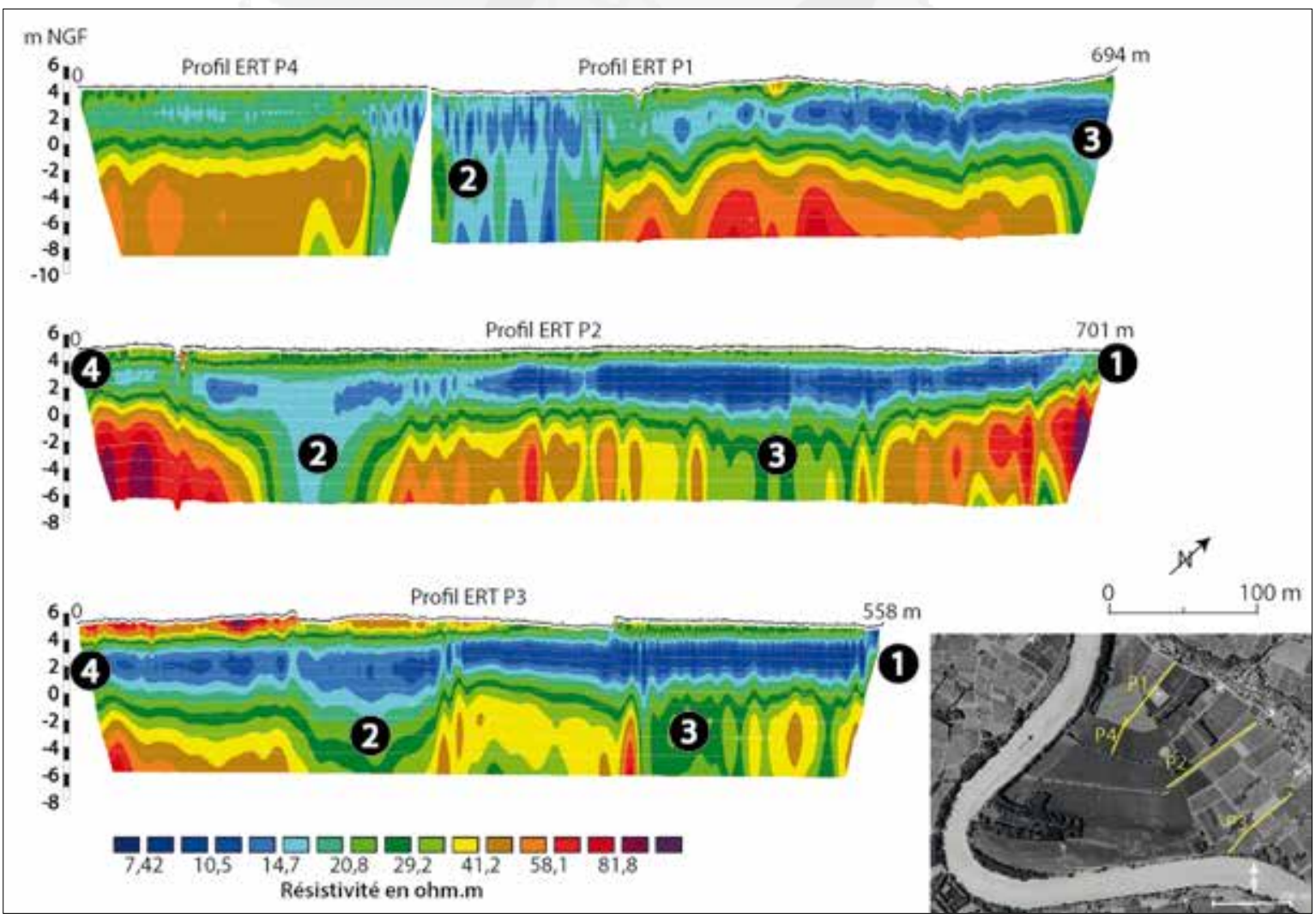

Fig. 11. Profils de résistivité électrique dans le secteur de Langoiran. (c) Lescure, 2014.

1. Remontée de résistivité correspondant potentiellement à une berge ; 2 . Paléochenal actif à la fin du Mésolithique ou antérieur ; 3. Paléochenal actif à la fin du Mésolithique ; 4. Autre paléochenal ? 
Le scénario 2 sous-tend l'idée de plusieurs paléo-bras fluviaux séparés par des îles ou des bancs alluviaux. La petite éminence topographique sur laquelle repose le Castéra de Langoiran pourrait correspondre à un banc alluvial au vu de sa granularité (sables) et de l'absence de sols pédologiques - régulièrement remaniés par les écoulements fluviaux, les bancs alluviaux n'ont pas le temps d'être fixés par un dense couvert végétal, contrairement aux îles fluviales. Suite à la remontée holocène du niveau marin, le fleuve a réduit sa pente pour rejoindre son nouveau niveau de base $\left(-5 \mathrm{~m}\right.$ NGF tout au plus à 6000 a.C. $\left.{ }^{21}\right)$. Un exhaussement du plancher alluvial se produit alors par abandon d'une partie de la charge solide, ce qui a pu former des bouchons de remblaiement dans certains paléochenaux condamnés à devenir des bras-morts marécageux, tandis qu'un seul et unique chenal d'écoulement a perduré au Néolithique. Un scénario similaire a été démontré dans la vallée de la Loire ${ }^{22}$.

\section{Aux âges des Métaux}

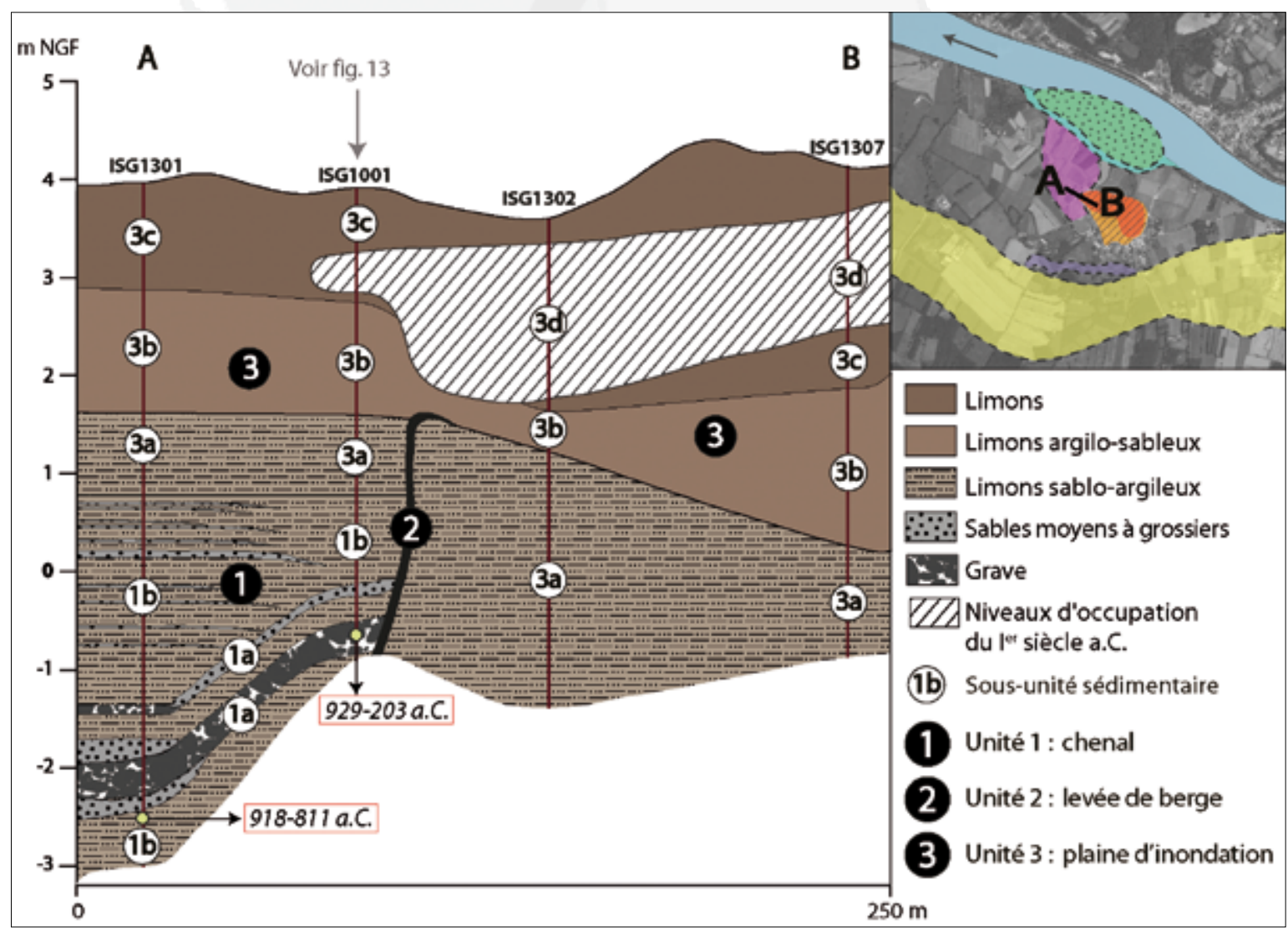

Fig. 12. Transect stratigraphique à travers le paléochenal contemporain de l'occupation protohistorique d'Isle-Saint-Georges. (C) Lescure, 2014. Deux niveaux grossiers (1a) sont intercalés entre un niveau plus fin (1b), indiquant probablement une phase de réactivation du chenal.

21- Pirazzoli 1991.

22. Carcaud 2004. 
Un chenal aurait été actif à l'ouest de la zone d'occupation protohistorique d'Isle-Saint-Georges (fig. 7 B) jusqu'au premier ou au Second âge du Fer (hypothèse étayée par deux dates radiocarbones que nous avons réalisées).

Sa présence a été décelée grâce à une forte conductivité électrique ${ }^{23}$ et confirmée par la présence de grave $^{24}$. Le transect stratigraphique réalisé dans le secteur a permis de localiser l'une des berges à l'extrémité occidentale de la zone d'habitat et de comprendre la dynamique du chenal (fig. 12). Ce dernier aurait connu différentes phases d'activité durant les âges des Métaux, comme en témoigne l'alternance de niveaux sableux avec des niveaux graveleux ou limono-sableux. Puis il aurait été progressivement abandonné au profit d'un autre bras (défluviation) ou par migration latérale, puisque la stratigraphie démontre un granoclassement vertical normal (grains de plus en plus fins) de la dernière unité de grave jusqu'aux limons argileux en surface

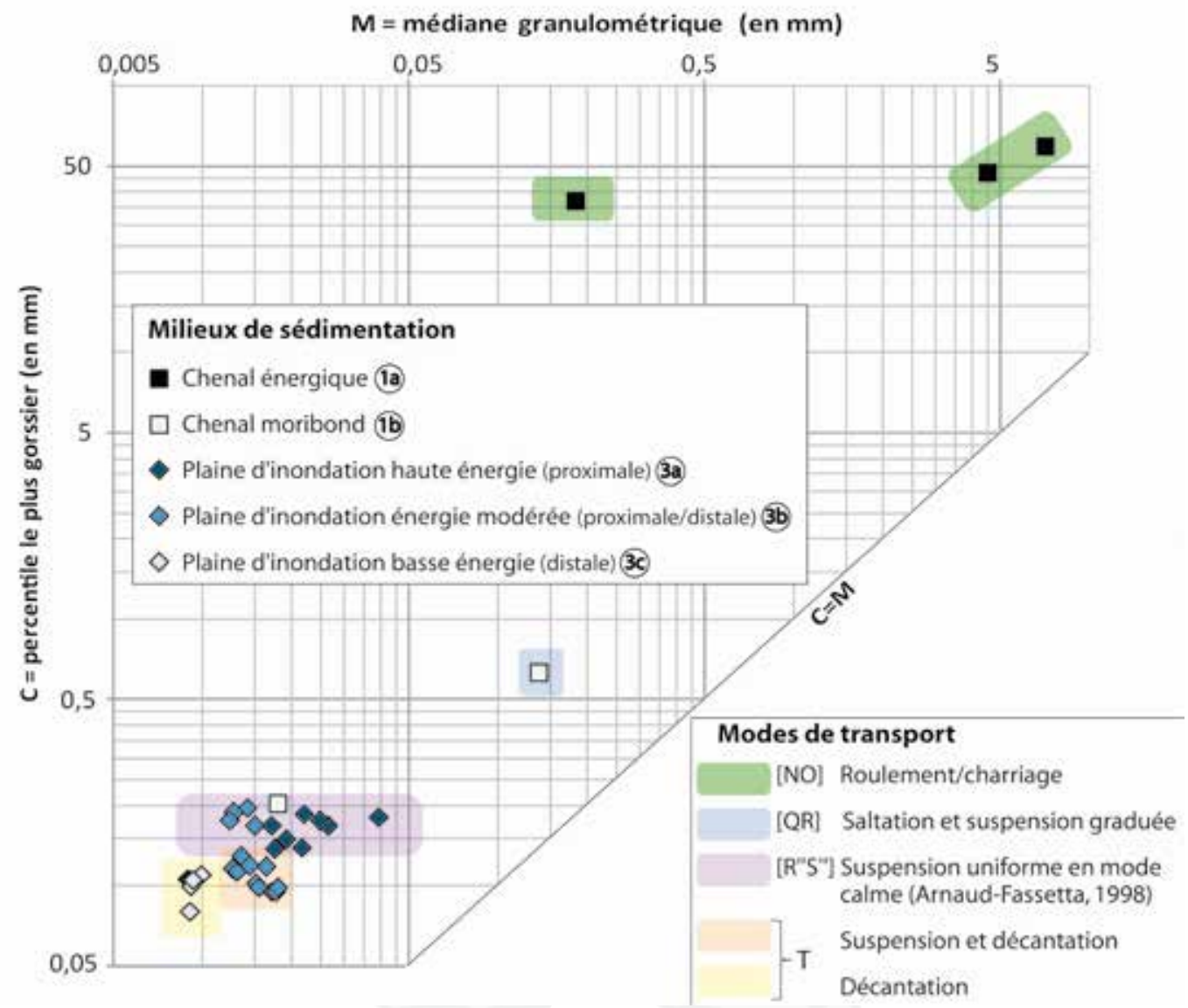

Fig. 13. Image CM de la séquence ISG1001 prélevée dans la berge du paléochenal contemporain de l'occupation protohistorique d'Isle-Saint-Georges.

D'après Lescure, 2011, modifié. Le diagramme de Passega, qui met en relation la compétence maximale (C) avec la compétence moyenne (M) d'un écoulement, permet ici d'identifier 1) un chenal énergique, qui transporte une charge supérieure à $50 \mathrm{~mm}$ par roulement et charriage, 2) un chenal moribond, qui véhicule par saltation et suspension des sédiments n'excédant pas 0,6 mm, et 3) une plaine d'inondation sur laquelle des suspensions fines se déposent par décantation.

23. Mathé \& Camus dans ce volume.

24- Lescure 2011, 38-47. 
(fig. 12). Nécessitant une énergie importante pour être transportés, la grave et les sables sont associés à des dépôts de chenal alors que les sédiments plus fins (sables-argileux, limons sableux, limons) sont interprétés comme des dépôts de plaine d'inondation. La persistance d'un granoclassement vertical au sein des dépôts d'inondation traduit soit une perte d'intensité des crues, soit un éloignement du cours d'eau à l'origine des inondations. Cette interprétation hydrologique des archives sédimentaires est corroborée par l'image $\mathrm{CM}^{25}$ construite à partir des échantillons prélevés dans les dépôts de la levée de berge supposée (fig. 13). Celle-ci fait état du passage d'un chenal énergique transportant sa charge par roulement / charriage à un chenal moribond véhiculant les sédiments par saltation et suspension, puis à une plaine d'inondation à énergie graduellement décroissante (décantation de suspensions fines).

\section{De la fin de l'Antiquité à nos jours}

Jusqu'à l'Antiquité tardive, voire au début du Moyen Âge, un cours d'eau semble avoir traversé le secteur sud-ouest du bourg d'Isle-Saint-Georges (fig. 7 B). Les données géophysiques laissent penser que ce paléochenal était plutôt un bras secondaire de la Garonne (70 à $100 \mathrm{~m}$ de largeur d'après les prospections

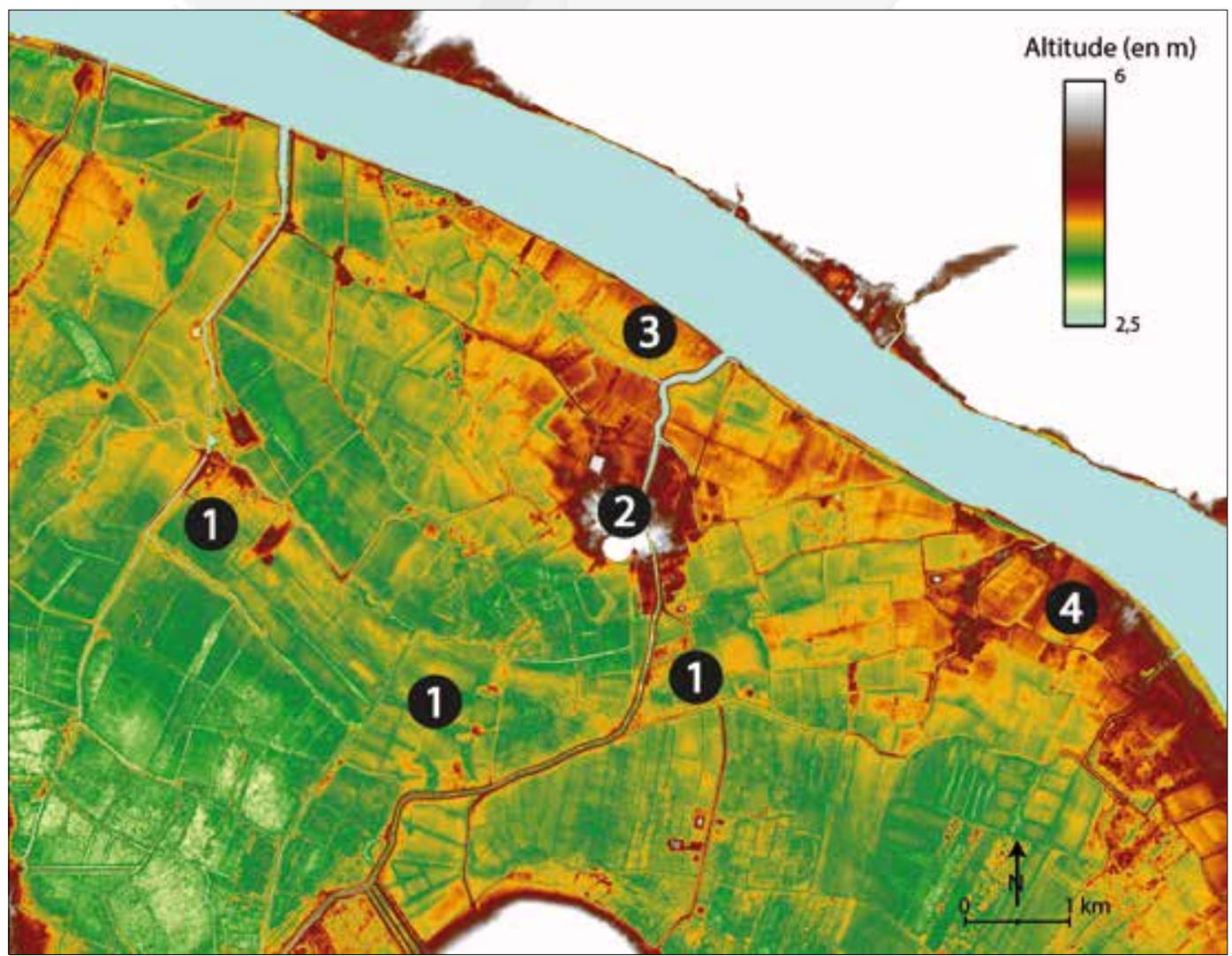

Fig. 14. Microtopographie du secteur d'Isle-Saint-Georges. (C) Lescure, 2014. La microtopographie a été mise en évidence par le traitement des données LIDAR. À Isle-Saint-Georges, quatre éléments légèrement surélevés sont mis en évidence : 1) le paléochenal mésolithique, 2) le bourg et la motte médiévale, 3) l'emplacement supposé de l'île attestée au xIII' $s$. et 4) l'atterrissement formé naturellement en rive convexe (dépôt d'alluvions car le courant y est plus faible). 
électromagnétiques et pas d'empreinte notable dans les profils ERT). Néanmoins, il était suffisamment énergique pour transporter des galets et des sables (datés à leur base de 416-557 p.C.).

L'étude n'a pas permis d'identifier de paléo-tracés plus récents à Isle-Saint-Georges mais la mention d'une île au XIII ${ }^{e}$ s. entre Isle-Saint-Georges et Cambes, soit en rive droite ${ }^{26}$, atteste une modification du lit fluvial du Moyen Âge à nos jours. Cette île se situait potentiellement au nord du bourg, au vu du parcellaire et de la topographie (fig. 14).

À Langoiran, aucun paléochenal contemporain de l'occupation du Castéra n'a été mis en évidence par le couplage stratigraphie/datations, les dépôts de sables et de grave présents sous et/ou à proximité du site étant tous d'âge néolithique. Il est néanmoins probable que la Garonne était plus proche du Castéra durant son occupation qu'elle ne l'est de nos jours puisque, comme le démontrent les cartes anciennes, le méandre décrit par le fleuve dans ce secteur n'a cessé de migrer vers l'aval depuis au moins le XVIII s. (fig. 7 A).

\section{Paléoenvironnements, contrainte fluviale et dynamique de l'occupation du sol}

\section{À Langoiran}

Le Castéra de Langoiran a été construit à une centaine de mètres seulement de la Garonne, alors en cours de migration, sur une entité sableuse fossile potentiellement héritée d'un banc alluvial d'âge néolithique.

Cette situation présentait des avantages certains pour les habitants (ressources vivrières, voie de communication) sans exclure de fortes contraintes puisque ces derniers étaient directement exposés aux crues débordantes du fleuve. La même logique/stratégie d'implantation semble avoir sous-tendu l'installation de petits établissements ruraux dans le delta du Rhône dès le ve s. a.C. ${ }^{27}$. Le fleuve Garonne peut-il donc avoir contribué à l'abandon du site de Langoiran, par le danger qu'il représentait en termes d'inondation et de changement de tracé ? Oui, si les sociétés ont connu une crue dévastatrice (type "crue du siècle") ou si le risque fluvial s'est accru (accroissement de l'intensité et/ou de la fréquence des crues et/ou des étiages; recul de la berge). En outre, il est probable que l'élévation de la plate-forme au cours du XIII $\mathrm{s}$. visait à protéger les occupants du castrum des inondations, mais cette action n'a pas été suffisante face à un risque croissant. Néanmoins, à ce jour, ni les données environnementales, ni les données historiques ${ }^{28}$ n'indiquent un tel accroissement du risque hydrologique. Le rôle direct du fleuve (aléa) dans l'abandon du site (vulnérabilité des enjeux et faible résilience des populations) n'est donc pas attesté ici.

En revanche, l'humidité du milieu (c'est-à-dire l'hydromorphie) pourrait avoir contribué à la désertion du Castéra. Durant son occupation, ce dernier était bordé par une zone humide, au moins à l'ouest. La toponymie en a archivé le souvenir (lieu-dit "la palus du Gardera") et l'étude ostracologique le démontre (présence de Candona compressa, espèce d'eau douce stagnante, dans les niveaux probablement contemporains du site ; fig. 9 C). Les données ne permettent pas de préciser la chronologie de mise en place de cette zone humide. Cependant, on peut envisager qu'elle se soit développée durant l'occupation du Castéra, dégradant potentiellement la qualité de vie. Par ailleurs, cette zone humide doit avoir favorisé la stagnation des eaux de débordement de la Garonne. Lors des crues moyennes à fortes, il est probable que les eaux stagnaient des jours durant dans la plaine d'inondation, isolant la "butte" du Castéra du reste de la vallée. Cela est 1) corrélable à la présence de matière organique et d'ostracodes d'eau stagnante dans les niveaux limoneux contemporains de l'occupation et 2) d'autant plus envisageable au vu de la nappe phréatique sub-affleurante

26- Ducaunnès-Duval 1892, 96.

27- Arnaud-Fassetta \& Landuré 2003.

28. Champion 1862 . 
(battement de la nappe perçu par l'oxydation des sédiments dès 1,50 $\mathrm{m}$ de profondeur et remontée de l'eau en surface dès $\sim 3 \mathrm{~m}$ de carottage) et en cas de forts coefficients de marée.

\section{À Isle-Saint-Georges}

Les sociétés protohistoriques d'Isle-Saint-Georges se sont vraisemblablement installées dans la plaine d'inondation proximale de la Garonne.

On ne sait pas encore si le fleuve passait uniquement à l'ouest de la zone d'occupation ou si cette dernière était en position insulaire, ce qui expliquerait alors la toponymie de la commune. Là encore, une telle proximité présentait des avantages indéniables et des contraintes fortes, les sociétés étant exposées à tout type d'inondation du fleuve.

Aucun élément n'indique l'occurrence d'une crue exceptionnelle ou un accroissement du risque fluvial durant l'occupation. Cependant, le repli de l'habitat entre le IV ${ }^{\mathrm{e}}$ s. et le II ${ }^{\mathrm{e}}$ s. a.C. pourrait concorder avec la fin de l'activité du chenal ou, tout du moins, avec une phase de moindre activité (fig. 12). L'arrêt de fonctionnement du chenal mettant fin aux activités fluviales (pêche, commerce), l'habitat a pu alors 1) se redéployer ailleurs (au bord d'un autre chenal toujours actif) ou 2) simplement s'éloigner de cette zone humide, dont l'intérêt devenait insuffisant en regard des contraintes qu'elle engendrait.

\section{CONCLUSION}

Les sociétés anciennes se sont installées dans la plaine d'inondation de la Garonne, à moins de $100 \mathrm{~m}$ de cette dernière. Une telle proximité au fleuve les exposait à un risque qu'elles n'ignoraient sûrement pas mais qui devait leur paraître secondaire face aux avantages que présentait cette situation (pêche, commerce fluvial, ressource en eau douce). L'étude n'a pas mis en lumière d'événement hydrologique extrême ou un accroissement de la contrainte fluviale qui pourrait expliquer l'abandon total (Castéra) ou partiel (Isle-SaintGeorges) des sites. Néanmoins, le site emmoté du Castéra de Langoiran était à la fois situé à proximité d'un fleuve en migration, bordé d'une zone humide et probablement coupé du reste de la vallée lors d'inondations modérées à fortes. De telles contraintes peuvent tout à fait avoir conduit à l'abandon du site en faveur d'un lieu en hauteur non exposé aux variations spatiales et hydrologiques du fleuve et dépourvu d'hydromorphie. Mais en l'absence de connaissances sur les dynamiques socio-politiques régionales, cette "répulsivité" potentielle du milieu ne peut être pleinement appréciée. Il en est de même à Isle-Saint-Georges où la contraction de l'habitat pourrait être liée à la fin de l'activité hydrologique du chenal adjacent. Des investigations supplémentaires, notamment chronologiques, seraient nécessaires pour conforter cette hypothèse. Ceci est d'autant plus nécessaire que les données environnementales acquises démontrent une très grande complexité de l'histoire holocène de la Garonne maritime dans ce secteur avec, notamment, l'occurrence de chenaux multiples au Mésolithique.

\section{Bibliographie}

Araguas, P. et S. Faravel (2006) : "Les châteaux de Langoiran, premier bilan archéologique", in : Cocula \& Combet, éd. 2006, 249-263.

Arnaud, F., M. Revel, E. Chapron, M. Desmet et N. Tribovillard (2005): "7200 Years of Rhône River Flooding Activity in Lake Le Bourget, France: a High-resolution Sediment Record of NW Alps Hydrology", The Holocene, 15, 3, 420-428.

Arnaud-Fassetta, G. (1998) : Dynamiques fluviales holocènes dans le delta du Rhône, Thèse de Géographie, université d'AixMarseille 1.
— (2008) : "La géoarchéologie fluviale : concepts, attendus et méthodes d'étude rétrospectives appliqués à la caractérisation du risque hydrologique en domaine méditerranéen", Échogéo [En ligne], 4, $10 \mathrm{p}$.

Arnaud-Fassetta, G. et L. Chabal (1997) : "Évolution des paléoenvironnements fluviaux dans la plaine deltaïque du Rhône de l'Antiquité au Haut Moyen-Age d'après la géomorphologie et l'anthracologie", in : Baudat, éd. 1997, 13-29.

Arnaud-Fassetta, G. et C. Landuré (2003) : "Hydroclimatic hazards, vulnerability of societies and fluvial risk in the Rhône Delta 
(Mediterranean France) from the Greek period to the Early Middle Ages", in : Fouache, éd. 2003, 51-76.

Arnaud-Fassetta, G. et N. Carcaud, éd. (2015) : French Geoarchaeology in the 21st century, Paris.

Arnaud-Fassetta, G., H. Bruneton, J.-F. Berger, C. Beaudouin, $X$. Boès et $M$. Provansal (2005) : "A 8000-yr record of palaeohydrology and environmental change in fluviatileinfluenced sediments from the urban Arles-Piton core, upper Rhône Delta, France", Zeitschrift für Geomorphologie, 49, 4, 455-484.

Baudat M., éd. (1997) : Crau, Alpilles et Camargue. Histoire et archéologie, Actes du colloque des 18 et 19 novembre 1995, Groupe archéologique arlésien, Arles.

Bravard, J.-P. (1983) : "Les sédiments fins des plaines d'inondation dans la vallée du Haut-Rhône (approche qualitative et spatiale)", Revue de Géographie Alpine, 71, 4, 363-379.

Bravard, J.-P. et F. Petit (1997) : Les cours d'eau, dynamique du système fluvial, Paris.

Bravard, J.-P., C. Amoros et C. Jacquet (1986) : "Reconstitution de l'environnement des sites archéologiques fluviaux par une méthode interdisciplinaire associant la géomorphologie, la zoologie et l'écologie", Revue d'archéométrie, 10, 43-55.

Bronk Ramsey, C. (1995) : "Radiocarbon calibration and analysis of stratigraphy: The OxCal program", Radiocarbon, 37, 2, 425430.

Buzzi, P., N. Carcaud, M.-P. Koenig et A. Weisrock (1993) : "Morphodynamique fluviale holocène et établissements humains protohistoriques en fond de vallée de la Moselle à Crévéchamps (Lorraine méridionale) ", Revue géographique de I'Est, 4, 281-295.

Carcaud, N. (2004) : D'espace et de temps : un itinéraire de recherche et d'enseignement sur les anthroposystèmes fluviaux, mémoire d'habilitation à diriger les recherches, Université d'Angers.

Castanet, C. (2008) : La Loire en val d'Orléans. Dynamiques fluviales et socio-environnementales durant les derniers 30000 ans : de l'hydrosystème à l'anthroposystème, thèse de Doctorat, Université Paris 1 - Panthéon-Sorbonne.

Castanet, C., C. Camerlynck, M.A. Vella, M. Garcin et J. Burnouf. (2007) : "Approche géophysique, sédimentologique et géomorphologique intégrée pour la caractérisation des dynamiques fluviales de la Loire survenues durant les derniers 25000 ans (Val d'Orléans, Loiret)", in : Actes du $6^{e}$ colloque GEOFCAN, Bondy, France, 99-102.

Champion, M. (1862) : Les inondations en France depuis le ve siècle jusqu'à nos jours, tome 4, Paris.

Clavé, B. (2001) : Évolution des paléo-environnements côtiers à I'Holocène : exemple de l'Aquitaine Septentrionale, thèse de Doctorat d'Océanographie et paléo-hydrographie, Université Bordeaux 1.

Cocula A.-M. et Combet M., éd. (2006) : Châteaux, livres et manuscrit, $1 x^{e}-x x^{e}$ siècles: Actes des rencontres d'archéologie et d'histoire en Périgord, Scripta Mediævalia Ausonius, Bordeaux.

Dean, W.E. (1974) : "Determination of carbonate and organic matter in calcareous sediments and sedimentary rocks by loss on ignition: comparison with other methods", Journal of Sedimentary Petrology, 44, 242-248.

Dearing, J.A., K.L. Hay, S.M.J. Baban, A.S. Huddleston, E.M.H. Wellington et P.J. Loveland (1996) : "Magnetic susceptibility of soil: an evaluation of conflicting theories using a national data set", Geophysical Journal International, 127, 728-734.

Diot, M.-F. et J.-P. Tastet (1995) : "Paléo-environnements holocènes et limites chrono-climatiques enregistrés dans un marais estuarien de la Gironde (France)", Quaternaire, 6, 2, 63-75.

Ducaunnès-Duval, A., éd. (1892) : Cartulaire de l'abbaye SainteCroix de Bordeaux (Archives Historiques de la Gironde, XXVII), Bordeaux,

Faravel, S. (2008) : Le site du Castéra et les palus de Langoiran : un site castral médiéval et son milieu, Rapport d'activités 2007, SRA Aquitaine.

- (2010) : Le castrum du Castéra (commune de Langoiran, Gironde), Rapport de fouille programmée 2008, SRA Aquitaine.

- (2012) : Le castrum du Castéra (commune de Langoiran, Gironde), Rapport de fouille programmée 2010, SRA Aquitaine.

- (2013) : Castrum du Castéra (commune de Langoiran, Gironde), Rapport de fouille programmée 2012, SRA Aquitaine.

Fouache, É., éd. (2003) : The Mediterranean World Environment and History, Paris.

Frouin, M., D. Sebag, A. Durand et B. Laignel (2010) : "Palaeoenvironmental evolution of the Seine River estuary during the Holocene", Quaternaire, 21, 1, 71-83.

Gé, T., W. Migeon et B. Szepertyski (2005) : "L'élévation séculaire des berges antiques et médiévales de Bordeaux. Étude géoarchéologique et dendrochronologique", Comptes rendus Géoscience, 337, 297-303.

Ghilardi, M., Y. Tristant et M. Boraik (2012) : "Nile River evolution in Upper Egypt during the Holocene: palaeoenvironmental implications for the Pharaonic sites of Karnak and Coptos", Géomorphologie : relief, processus, environnement, 1, 7-22.

Heiri, O., A.F. Lotter, et G. Lemcke (2001) : "Loss on ignition as a method for estimating organic and carbonate content in sediments: reproducibility and comparability of results", Journal of Paleolimnology, 25, 101-110.

Konik, S., G. Allenet, P. Carbonel, B. Clavé, A. Guilhou, V. Guitton, C. Leroyer et J.-P. Tastet (2006) : Évolution paléoenvironnementale des berges de la Garonne, paysages végétaux et étapes de l'anthropisation du site de Bordeaux, Rapport de fouilles préventives, Inrap, SRA Aquitaine.

Lane, E.W. (1955) : "The importance of fluvial morphology in hydrologic engineering", Proceedings American Society of Civil Engineers, 81, 1-17.

Lejeune, Y., C. Leroyer et J.-F. Pastre (2012) : “L'évolution holocène de la basse vallée de la Marne (Bassin parisien, France) entre influences climatiques et anthropiques", Géomorphologie : relief, processus, environnement, 4, 459-476.

Lescure, S. (2011) : Reconstitutions des paléoenvironnements fluviaux dans la basse vallée de la Garonne: le cas de l'IsleSaint-Georges, Mémoire de master 2 de Géographie, Université Paris-Est Créteil.

Macklin, M.G. (1999) : "Holocene river environments in prehistoric Britain: human interaction and impact", Journal of Quaternary Science, 14, 521-530.

Malounguila-Nganga, D., J. Nguie et P. Giresse (1990) : "Les paléoenvironnements quaternaires du colmatage de l'estuaire du Kouilou (Congo)", in : Paysages quaternaires de l'Afrique centrale atlantique, ORSTOM, Paris, 89-97. 
Passega, R. (1964) : "Grain size representation by CM patterns as a geologic tool", Journal of Sedimentary Petrology, 34, 830-847.

Pirazzoli, P.A. (1991) : World Atlas of Holocene sea-level changes, Elsevier Oceanography Series, Amsterdam.

Pontee, N.I., J.-P. Tastet et L. Masse (1998) : "Morpho-sedimentary evidence of Holocene coastal changes near the mouth of the Gironde and on the Medoc Peninsula, SW France", Oceanologica Acta, 21, 2, 243-261.

Qinghai, X., W. Chen, Z. Xuanqing et Y. Xiaolian (1996) : "Palaeochannels on the North China Plain, stage division and palaeoenvironments", Geomorphology, 18, 15-25.

Salomon, F. (2013) : Géoarchéologie du delta du Tibre - Évolution géomorphologique holocène et contraintes hydrosédimentaires dans le système système Ostie-Portus (Italie), thèse de Doctorat en Géographie, Université Lumière Lyon 2.

Salvador, P.-G., G. Arnaud-Fassetta, N. Carcaud, C. Castanet et L. Ferdinand (2009) : "Dynamique fluviale holocène et géoarchéologie en milieu fluviale", in : Les géographies de
I'eau. Processus, dynamique et gestion de l'hydrosystème, Paris, 181-228.

Schumm, S. A. (1977) : The fluvial system, New York.

Thompson, R. et D.J. Morton (1979) : "Magnetic susceptibility and particle-size distributions in recent sediments of the Loch Lomond drainage basin, Scotland", Journal of Sedimentary Petrolology, 49, 801-811.

Vigreux, T., D. Aoustin, J.-P. Degeai et A. Koziol (2012) : "Évolution de la plaine alluviale du Rhin dans la région du "Ried nord" : paléoenvironnement et interactions anthropiques depuis l'Âge du Bronze jusqu'à l'Antiquité/Haut Moyen Âge (Roeschwoog, Bas-Rhin, Alsace)", Quaternaire, 23, 4, 321-337.

Waters, M.R. (1988) : "Holocene alluvial geology and geoarchaeology of the San Xavier reach of the Santa Cruz River, Arizona", Geological Society of America Bulletin, 100, 4, 479-491.

Whatley, R.C. (1988) : "Ostracoda and palaeogeography", Ostracoda in the earth sciences, 103-123. 
